\title{
REVIEW ARTICLE OPEN Inorganic semiconducting materials for flexible and stretchable electronics
}

\author{
Ki Jun $\mathrm{Yu}^{1}$, Zheng $\mathrm{Yan}^{2}$, Mengdi $\mathrm{Han}^{3}$ and John A. Rogers ${ }^{4}$
}

Recent progress in the synthesis and deterministic assembly of advanced classes of single crystalline inorganic semiconductor nanomaterial establishes a foundation for high-performance electronics on bendable, and even elastomeric, substrates. The results allow for classes of systems with capabilities that cannot be reproduced using conventional wafer-based technologies. Specifically, electronic devices that rely on the unusual shapes/forms/constructs of such semiconductors can offer mechanical properties, such as flexibility and stretchability, traditionally believed to be accessible only via comparatively low-performance organic materials, with superior operational features due to their excellent charge transport characteristics. Specifically, these approaches allow integration of high-performance electronic functionality onto various curvilinear shapes, with linear elastic mechanical responses to large strain deformations, of particular relevance in bio-integrated devices and bio-inspired designs. This review summarizes some recent progress in flexible electronics based on inorganic semiconductor nanomaterials, the key associated design strategies and examples of device components and modules with utility in biomedicine.

npj Flexible Electronics (2017)1:4 ; doi:10.1038/s41528-017-0003-z

\section{INTRODUCTION}

Substantial interest exists in materials and fabrication techniques that enable electronic devices to be formed, in scalable ways and with high-performance operation, directly on flexible metal foils or plastic sheets. ${ }^{1-6}$ Recent additional, related efforts seek to establish capabilities in rendering electronics capable not only of bending but also of stretching, where elastomers serve as the substrates. $^{7-17}$ Such systems can establish intimate, conformal contacts to complex curvilinear surfaces, such as those found in biology, with endurance to high levels of strain. Neither such characteristics is possible with conventional, rigid technologies formed on the surfaces of semiconductor wafers or glass panels. Flexible electronic devices offer great potential for application in areas such as wearable electronics, ${ }^{2}, 8,11,18,19$ paper-like displays, $^{13,20-22}$ and health-monitoring systems. ${ }^{19,} 23-26{ }^{\text {pape }}$

Inorganic semiconductors represent the foundations for all conventional, commercialized types of electronic devices, due primarily to performance characteristics that significantly exceed those of known organic materials, including high-field-effect mobilities and long-term stability under mechanical, electrical, and environmental stress. ${ }^{27-30} \mathrm{~A}$ main challenge in the use of inorganic semiconductors and associated traditional processing methods follows from limitations in materials choices and fabrication strategies. For example, most polymer substrates are incompatible with the high temperatures required in traditional procedures for deposition, crystallization, and doping. Furthermore, manufacturing by roll-to-roll printing, over large areas and at low cost per unit areas can, in some cases, be more easily achieved with organic active materials than inorganic counterparts. Recent research ${ }^{7}, 9,31,32$ demonstrates, however, that suitable choices of materials, design layouts, and integration strategies allow use of the highest performance, monocrystalline inorganic semiconductors in mechanically compliant systems, in ways that overcome such limitations. Here, ultrathin, typically nanoscale, semiconductor material structures allow heterogeneous integration onto plastic or rubber substrates, with all other necessary materials to achieve sophisticated electronic function. The key factors that underpin mechanical flexibility in such cases include (i) downscaling of the flexural rigidity with the cube of the thickness of the structure, and (ii) reduction of the bending induced strain by use of neutral mechanical plane designs. ${ }^{33,} 34$

The most effective approaches to the realization of such systems involve growth and formation of inorganic semiconductor micro/nanostructures, sometimes involving high temperatures and chemically harsh conditions on appropriate substrates, followed by their deterministic assembly onto surfaces of interest at temperatures compatible with polymer supports. Here, the critical electronic materials properties can be optimized in a way that is decoupled from considerations associated with the final device substrates. The results are classes of flexible electronic systems with performance that can reach levels comparable to those of conventional wafer-based technologies, far exceeding the range accessible to known organic electronic alternatives. This review briefly summarizes some recent progress in this field, and highlights several device examples.

\footnotetext{
${ }^{1}$ School of Electrical and Electronic Engineering, Yonsei University, Seoul 03722, Republic of Korea; ${ }^{2}$ Department of Chemical Engineering and Department of Mechanical \& Aerospace Engineering, University of Missouri, Columbia, Missouri 65211, USA; ${ }^{3}$ Center for Bio-Integrated Electronics, Northwestern University, Evanston, Illinois 60208, USA and ${ }^{4}$ Departments of Materials Science and Engineering, Biomedical Engineering, Chemistry, Mechanical Engineering, Electrical Engineering and Computer Science, and Neurological

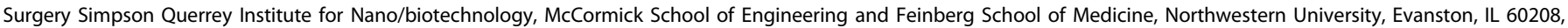
USA

Correspondence: Ki Jun Yu (kijunyu@yonsei.ac.kr) or John A. Rogers (jrogers@northwestern.edu)
}

Received: 5 January 2017 Revised: 20 March 2017 Accepted: 10 April 2017

Published online: 26 September 2017 


\section{BOTTOM-UP APPROACHES}

The required nanoscale inorganic semiconductor building blocks can be formed using chemical synthetic methods, sometimes referred to as "bottom-up" approaches, whereby controlled growth yields two-dimensional (2D) nanomembranes or nanoribbons (NMs, NRs), ${ }^{35}, 36$ one-dimensional (1D) nanowires (NWs), ${ }^{37}$ zero-dimensional (OD) nanoparticles, ${ }^{38}$ or in complex geometries that incorporate multiple such features. The most widely explored schemes involve NWs, where there are many examples of flexible/ stretchable electronics that exploit the outstanding electrical, ${ }^{39}$ mechanical, $^{40}$ and optical properties ${ }^{41}$ of these materials. In one representative case, hydro-thermal growth of vertically oriented $\mathrm{ZnO} \mathrm{NWs}$ yields active materials for adaptive tactile imaging systems, as shown in Fig. 1a. Here, the NWs electrically connect to top and bottom electrodes where they convert external mechanical stimuli into an electronic controlling signal that modulates the current flow by combining the semiconducting and piezoelectric properties of $\mathrm{ZnO}$. A sensing matrix built with $92 \times 92$ pixels that adopts this design demonstrates the scalability of this type of approach. Here, flexible and transparent substrates (i.e., sheets of polyethylene terephthalate) and transparent electrodes (indium tin oxide), and packaging materials (SU8 and parylene) are key components.

Compared with this case of direct growth of NWs on target device substrates, NWs formed on native substrates using optimized conditions, followed by assembly via physical transfer or other means, to desired substrates offer improved versatility in materials choices. In one such approach, ${ }^{42} \mathrm{NWs}$ of $\mathrm{Si}$ and Ge grown by the nanocluster-catalyzed vapour-liquid-solid method, can be integrated over large areas in highly aligned configurations by a nanoscale combing technique. Here, lithographically patterned surfaces on the target substrate provide anchoring regions (strong interfacial interactions) and aligning regions (weak interfacial interactions) to guide the process. On this basis, more

\section{* Bottom-up Approaches}
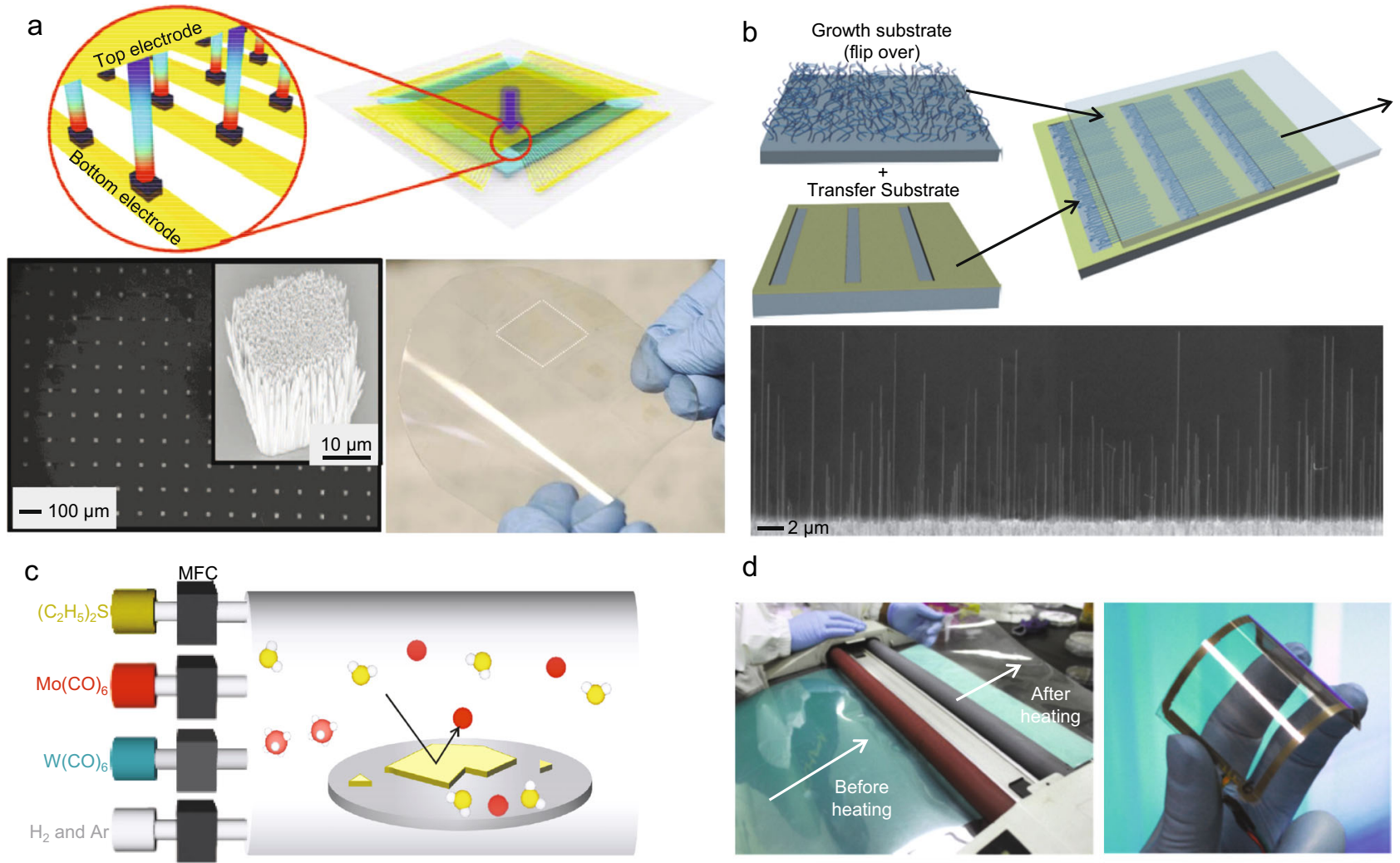

d
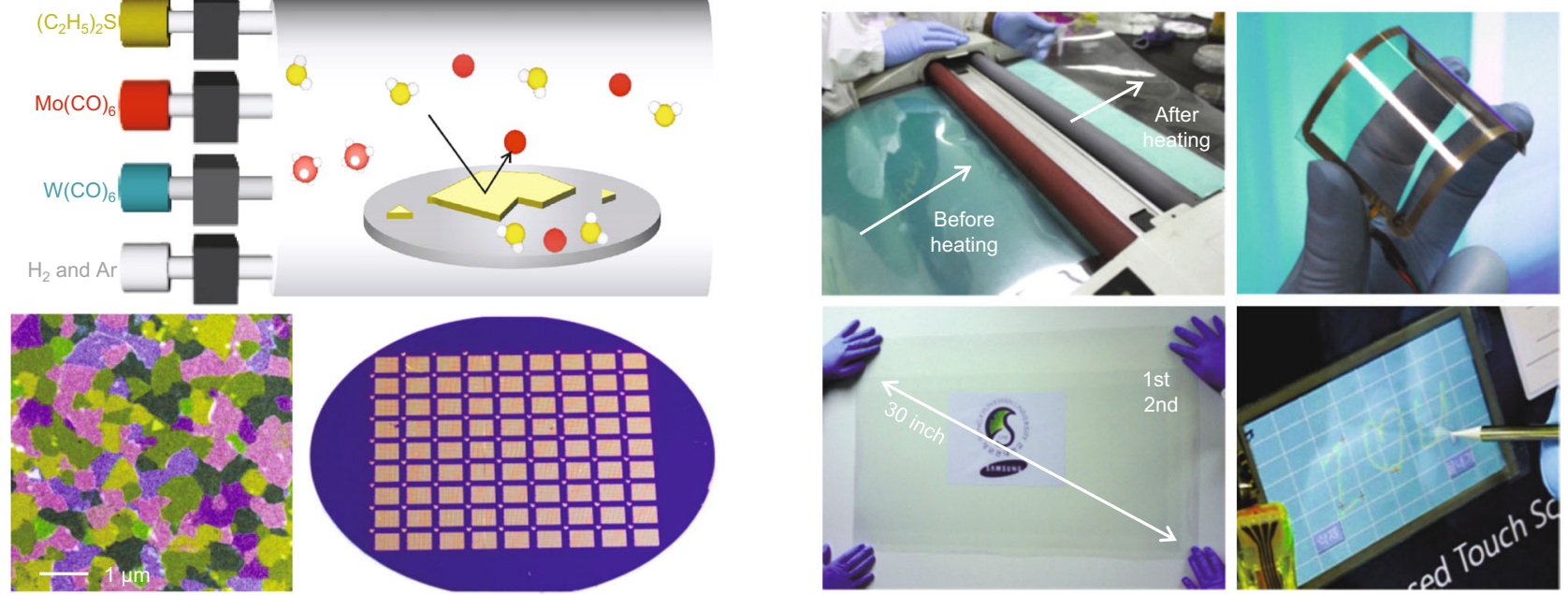

Fig. 1 Flexible and stretchable electronic devices that use monocrystalline semiconductor nanomaterials formed using synthetic, bottom-up approaches. a Flexible and transparent array of transistors that use vertically aligned ZnO NWs, configured into a system that allows large-area tactile imaging. Reprinted with permission from Wu et al. ${ }^{100}$ Copyright 2013 American Association for the Advancement of Science. b Ge/Si NW transistors formed by assembly of NWs using a nanoscale combing technique. Reprinted with permission from Yao et al. ${ }^{42}$ Copyright 2013 Nature Publishing Group. c Wafer-scale growth of monolayer $\mathrm{MoS}_{2}$ and $\mathrm{WS}_{2}$ for transistors that exhibit high mobilities. Reprinted with permission from Kang et al. ${ }^{48}$ Copyright 2015 Nature Publishing Group. d Roll-to-roll transfer of sheets of graphene as a possible path to commercialization for large-scale transparent and flexible electrodes. Reprinted with permission from Bae et al. ${ }^{50}$ Copyright 2010 Nature Publishing Group 
than $98.5 \%$ of the NWs can be assembled and aligned in the desired direction with less than $1^{\circ}$ deviation, where contact forces with the growth substrate, along with the directionality and speed of translation represent key variables, as shown in Fig. $1 \mathrm{~b} .{ }^{42}$ Large arrays of NW field effect transistors (FETs) are possible in this way, with excellent uniformity and reproducibility, as defined in one example by statistical characteristics of the performance of $\mathrm{Ge} / \mathrm{Si}$ NW transistors. Comprehensive reviews of NW-based flexible electronic technologies can be found in other articles. ${ }^{43,44}$

More recent work demonstrates the utility of 2D materials such as graphene and transition-metal dichalcogenides, ${ }^{45,}{ }^{46}$ as specific examples of NMs formed using the bottom-up concept. These and other 2D materials grown by chemical vapor deposition possess excellent electrical properties and can now be obtained in large scale formats, suitable for use in high-performance flexible electronic devices. ${ }^{47}$ Particularly promising results involve preparation of wafer-scale monolayers of $\mathrm{MoS}_{2}$ and $\mathrm{WS}_{2}$ through metal-organic chemical vapor deposition. ${ }^{48}$ As shown in Fig. 1C, gas-phase precursors of $\left(\mathrm{C}_{2} \mathrm{H}_{5}\right)_{2} \mathrm{~S}, \mathrm{Mo}(\mathrm{CO})_{6}, \mathrm{~W}(\mathrm{CO})_{6}$, and $\mathrm{H}_{2}$ yield highly uniform coverage of monolayers, and a layer-by-layer growth mode can be utilized at large scale. The continuity of the as-grown $\mathrm{MoS}_{2}$ film can be confirmed by darkfield transmission electron microscope and annular darkfield scanning transmission electron microscope images. Arrays of $\mathrm{MoS}_{2}$ and $\mathrm{WS}_{2}$ FETs show field-effect mobilities of $\sim 29$ and $18 \mathrm{~cm}^{2} \mathrm{~V}^{-1} \mathrm{~s}^{-1}$, respectively, suitable for many applications.

Capabilities in physical transfer of such as-grown 2D materials onto other substrates create opportunities in flexible electronics. ${ }^{49}$ Among various transfer printing techniques, a roll-to-roll process demonstrated with graphene films provides a compelling example with potential relevance at commercial scale for transparent conducting films. ${ }^{50}$ This scheme begins by passing a graphene film grown on a copper foil together with a polymer thin film between two rollers. After etching the copper with $0.1 \mathrm{M}\left(\mathrm{NH}_{4}\right)_{2} \mathrm{~S}_{2} \mathrm{O}_{8}$, the graphene can be released from the polymer by reducing the adhesion by thermal or other means, thereby allowing transfer to other substrates. As shown in Fig. 1d, graphene sheets over areas as large as 30-inch diagonal can be integrated onto plastic substrates, where they can be used as touch panels with outstanding transparency and flexibility. Similar schemes might be possible with semiconducting 2D materials and/or NMs as well. Additional information can be found in other reviews. ${ }^{51,52}$

\section{TOP-DOWN APPROACHES}

Alternatives to bottom-up approaches like those described in the previous section involve lithographic processing and etching to define semiconductor micro/nanomaterials from bulk or thin film sources of material with deterministic control over key dimensional characteristics and spatial arrangements. As one example of the former, defining trenches on the surfaces single crystalline silicon wafers with $<111\rangle$ orientation, and passivating the top surfaces and sidewalls prepare the substrate for anisotropic etching preferentially along the $\langle 110\rangle$ directions. ${ }^{3,53-55}$ Although most well explored for NRs, $53,54,56$ Fig. 2 shows an example of multiple cycles of this process, where each yields a single, waferscale NM, or a high-density collection of them. Figure 2a (left) schematically illustrates the fabrication steps. The process starts with photolithographic patterning of an etch resist to define the lateral dimensions of a collection of microbar structures that connect together in a manner that yields a continuous membrane but with perforations to allow access of etchant to underlying regions. Reactive ion etching of the exposed $\mathrm{Si}$ in a direction perpendicular to surface of the wafer (i.e., the $\mathrm{Si}\langle 110\rangle$ direction) defines the thicknesses of the bars. After passivating the top surface and sidewalls of these structures, immersion into a bath of $\mathrm{KOH}$ bath releases a wafer-scale NM by preferentially undercut etching the $\mathrm{Si}$ in the $\langle 110\rangle$ direction. Here, the NM remains tethered to the underlying wafer in an otherwise free-standing form. Membranes with thicknesses between $2 \mu \mathrm{m}$ to $30 \mu \mathrm{m}$, areal coverages of up to $90 \%$, depending upon the gap between microbars, and overall sizes up to $2 \times 2 \mathrm{~cm}$, possibly to the scale of the wafer itself, have been achieved. Related processes for NRs demonstrate the scalability of this process into sub-micron thickness regimes. ${ }^{53,54} \mathrm{NMs}$ formed in this way are exceptionally well configured for transfer onto flexible substrates by wellestablished methods of transfer printing., 57 The remaining source wafer can be reprocessed after chemically polishing the surface by immersion into $\mathrm{KOH}$, to yield additional generations of NMs, with the potential to convert most of the material of the wafer itself into NM form. The micrograph images in Fig. 2a (right) show an example that includes a third generation membrane.

Another approach, most applicable to NRs, involves control of the reactive ion etching process to yield well defined rippled sidewall morphologies, as described in the schematic illustration of Fig. $2 \mathrm{~b}$ (left). ${ }^{55}$ Here, angled physical vapor deposition of metal from a collimated source yields coatings selectively on the upper sides of these ripples. These coatings can serve as masks for anisotropic wet etching, as before, but in a way that releases multilayer stacks of ribbons or membranes in a single step. Figure $2 \mathrm{~b}$ (right) shows optical and scanning electron microscope (SEM) images of bulk quantities of Si NRs generated in this way.

A different class of top-down method uses embedded layers designed to be selectively eliminated to affect release. The most common approach uses silicon on insulator wafers ${ }^{58-60}$ (SOI), as illustrated in Fig. 2c (top). ${ }^{34}$ In this case, holes etched in the top silicon facilitate access to hydrofluoric acid for etching away the buried $\mathrm{SiO}_{2}$ layer, thereby releasing a layer of silicon in free standing form. The resulting Si NMs can be extremely flexible, as in Fig. 2c (bottom). ${ }^{37}$ Commercially available SOI wafers provide access to NMs with thicknesses down to approximately $20 \mathrm{~nm}$. Additional processing, based on sequential cycles of oxidation and etching, can yield ultrathin Si NMs, with thicknesses ranging from 1.4 to $10 \mathrm{~nm}^{61}$ Exceptionally thin, flexible devices are possible in this way, when combined with self-assembled monolayers, 2D materials and other ultrathin constructs. Transport properties can be influenced by the thicknesses in this regime. ${ }^{62}$

In advanced examples, Heteroepitaxial growth of a trilayer of $\mathrm{Si}$ / $\mathrm{SiGe} / \mathrm{Si} \mathrm{NMs}$ on an SOI wafer produces a lattice strain. Upon release, the compressive strain from $\mathrm{SiGe}$ layer redistributes towards the unstrained $\mathrm{Si}$, thereby creating controlled levels of strain in the NMs. ${ }^{63-65}$ Elastic strain relaxation from released NMs from SOI wafers provides an additional mechanism for control of both mobility and band offsets. ${ }^{63}$ Strain engineering in this manner may improve the characteristics of flexible devices formed with these materials. ${ }^{63,64}$ High-speed flexible electronic devices, some with operating frequencies that exceed $1 \mathrm{GHz}$, including MOSFETs, PIN diodes, single-pole single-throw switches can be realized with these and other Si NMs. ${ }^{66-68}$ By use of other SOl-like substrates, Ge NMs can be interesting for photodetection due to high-absorption over a wide range of wavelengths. ${ }^{69}$ Flexible arrays of PIN photodetectors fabricated with Ge NMs from germanium on insulator wafer (GeOI) offer excellent performance even after the severe bending. ${ }^{70}$

In another example, alternating stacks of gallium arsenide (GaAs) and aluminium gallium arsenide (AIGaAs) serve as the starting point for creating large quantities of GaAs NMs by selective removal of the AIGaAs with hydrofluoric acid. Figure $2 d^{71}$ shows an illustration of GaAs and AlGaAs stacks on a bulk GaAs wafer and release of GaAs NMs by selective HF etching. Similar multilayers can be produced in bulk quantities using this approach. Sacrificial layers of AlGaSb enable formation of ultrathin InA NMs; transfer printing allows integration with other substrates, to yield unique, heterogeneously integrated electronic devices. ${ }^{72}$ Purely inorganic interfaces with low-interface trap densities and high stability and with improved electrostatic coupling to the gate 


\section{Top-down Approaches}

a

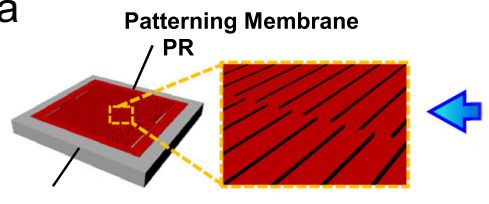

Si

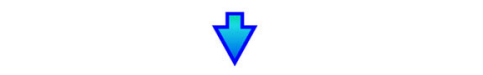

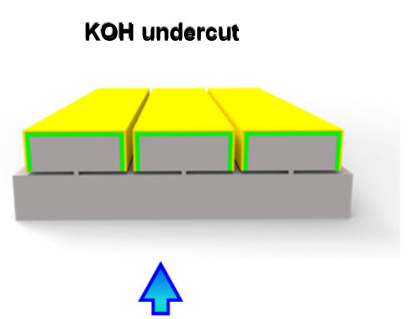

4

Define Trenches with ICP-RIE

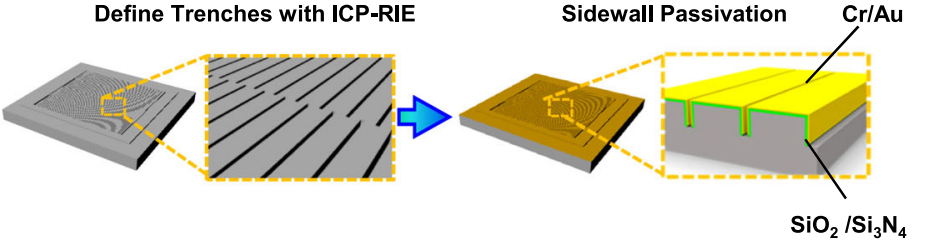

b
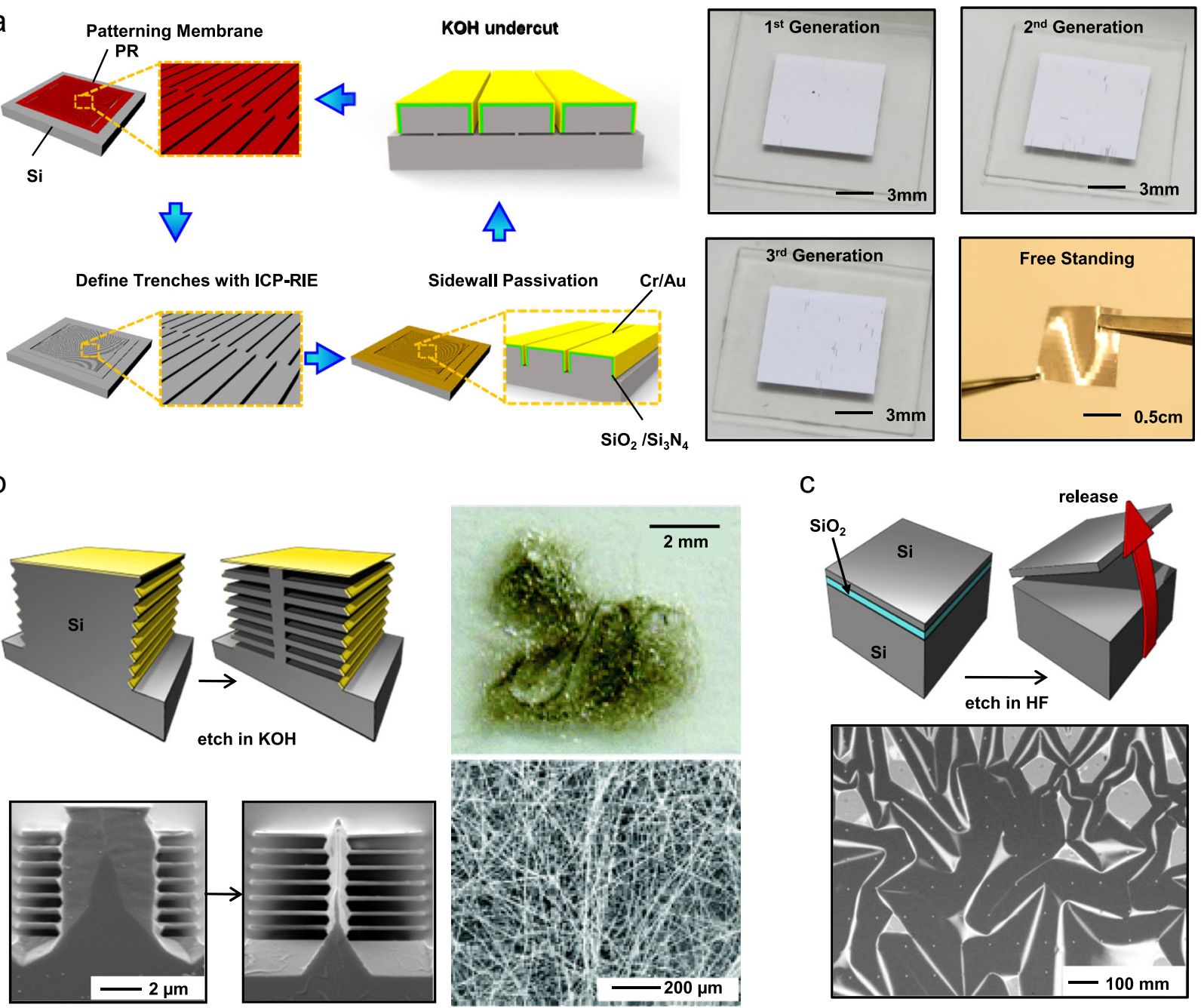

C
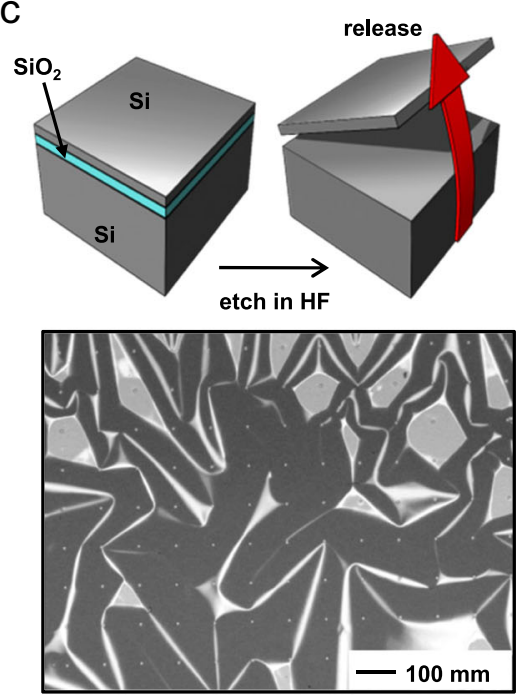

d
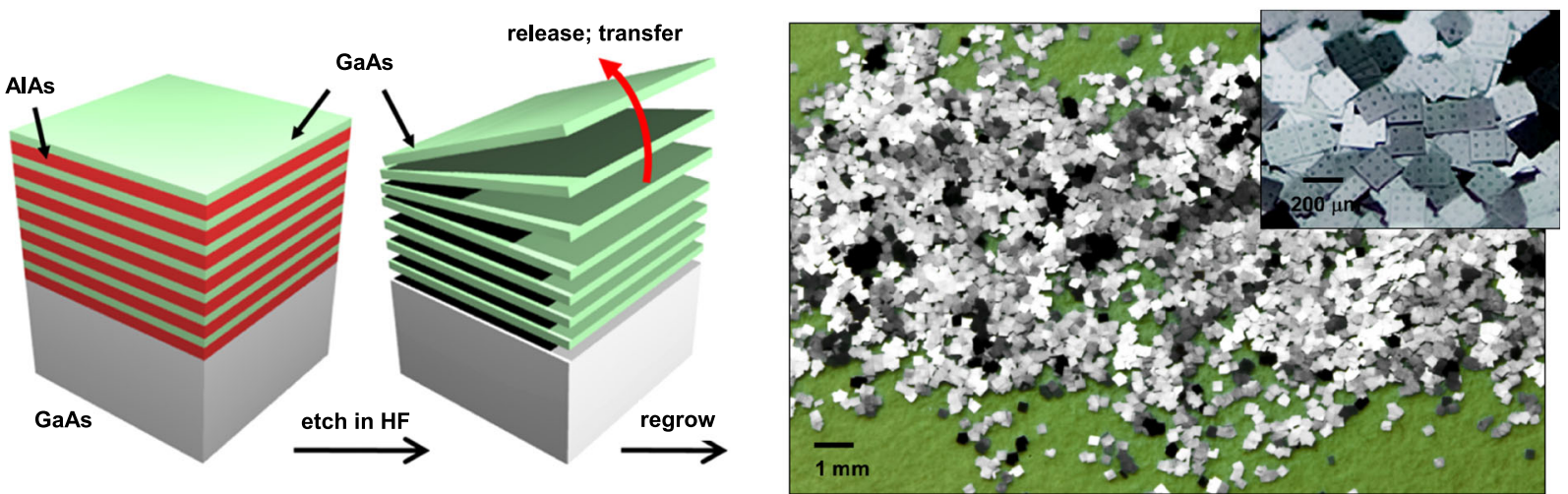

Fig. 2 Single crystalline inorganic nanomembranes and nanoribbons, formed using lithographically controlled, top-down approaches. a Flexible Si NM formed by anisotropic etching of a bulk wafer substrate (left). Repeated application of this process provides access to bulk quantities of Si NMs from a single wafer (right). $\mathbf{b}$ lllustration of a process for fabricating bulk quantities of Si NRs, using a multilayer stacked configuration, from a bulk wafer (left). Photograph and scanning electron micrographs of large quantities of NRs formed in this way (right). Reprinted with permission from Ko et al. ${ }^{55}$ Copyright 2006 American Chemical Society. c Illustration of the release of a Si NM from the device silicon layer on an SOI wafer (top). The optical image shows a wrinkled Si NM created in this manner. Reprinted with permission from Rogers et al ${ }^{34}$ Copyright 2011 Nature Publishing Group. d Illustration of multilayers of GaAs and AlAs grown on a bulk GaAs wafer. The optical image shows a collection of GaAs NMs that follow from selective removal of AlAs layers. Reprinted with permission from Yoon et al. ${ }^{11}$ Copyright 2010 Nature Publishing Group 
electrode led to superior switching properties. Additional unique possibilities follow from use of strained layers that lead to the spontaneous formation of tubular shapes in the released $\mathrm{NMs}^{73,74}$ These types of top-down methods offer advantages over bottomup schemes because they attempt to leverage, rather than displace, the most advanced methods in wafer and thin-film growth. Additionally, the engineering control over key structure dimensions and the lithographically defined spatial order and orientation lead to improved uniformity and yield in device fabrication. Multilayer methods such as those illustrated here can be crucially important for cost sensitive applications.

\section{MECHANICS}

Design approaches for flexible electronic systems that use the classes of inorganic semiconductor micro/nanostructures described in preceding sections rely mainly on thin geometries and neutral mechanical plane layouts. ${ }^{12}$ Theoretically, the flexural rigidity decreases with the cube of the thickness, which leads to more than fifteen orders of difference between Si NMs with thicknesses of $10 \mathrm{~nm}$ and bulk silicon wafers with thicknesses of 1 $\mathrm{mm}$. Figure 3a shows an example of bendability in Si NMs. ${ }^{34}$ Moreover, the bending strain associated with a given radius of curvature decreases linearly with the thickness, thereby providing an additional benefit associated with thin geometries. Additionally, the energy release rate associated with delamination between a film and a substrate also decreases linearly with the thickness, thereby facilitating the robust integration of NMs with substrates of dissimilar materials to form heterogeneous electronic systems. An example of the combined use of these concepts involves $\mathrm{Si}$ NRs transfer printed onto plastic sheets to form flexible thin-film transistors in Fig. 3b. ${ }^{53}$

Although mechanical flexibility can open up many applications that cannot be addressed with conventional rigid forms of electronics, stretchable characteristics provide a further level of distinction, with capabilities for conformal integration onto complex, non-developable surfaces, and the capacity to function under deformations that involve large strains. ${ }^{75}$ The first report of a stretchable form of an inorganic semiconductor exploited buckling of Si NRs on an elastomer substrate, to yield a "wavy" configuration. ${ }^{76}$ Here, the geometry of this hard/soft composite can change in response to applied strain, in a way that avoids significant strains in the silicon. One simple approach to form the necessary structure is to bond the Si NRs to a stretched elastomer, and then to release the stretch. This process leads to compressive stresses on the initially flat Si NRs to spontaneously transform them into highly periodic wavy shapes, as a result of a non-linear buckling instability, as shown in Fig. 3c. The amplitude and wavelength depend on the thickness of the silicon, and other key material properties.

An advanced approach that provides additional control over these critical geometrical parameters ${ }^{77}$ uses lithographic techniques to define sites of adhesion to the elastomer, typically by treating the native oxide surfaces of the semiconductors and a silicone elastomer to ultraviolet (UV) ozone to produce -OH termination. Condensation reactions initiated by physical contact yield strong bonding at these sites; other regions involve only weak van der Waals interactions. The latter delaminate and buckle upward, out of the plane, during release of the stretch in the substrate as shown in Fig. $3 \mathrm{~d}$. In this way, the geometries can be deterministically engineered to achieve significant increases in the range of stretchability. Additional options utilize not only out-ofplane but also in-plane buckling and bending. Here, the Si NRs take the form of filamentary serpentines. ${ }^{78}$ The most sophisticated embodiments exploit concepts of fractal mechanics in the designs, ${ }^{79}$ as space-filling curves that offer unique mechanical properties. Representative fractal-inspired layouts include von Koch, Peano and Hilbert curves, as well as Vicsek shapes. Figure 3e shows schematic illustrations of each of these possibilities, along with corresponding experimental microscale X-ray coherent tomography (MicroXCT) images after stretching.

\section{EPIDERMAL ELECTRONICS}

Assemblies of inorganic semiconductors on thin plastic sheets or elastomeric films can be utilized as active components for highperformance electronic sensing and/or actuating platforms with high degrees of mechanical bendability or deformability. Some of the most compelling applications for such classes of electronics are in systems that intimately interface with living organisms. In these cases, active inorganic materials with designs like those described previously embed in soft elastomeric matrices to serve as the basis for integrated collections of devices. ${ }^{8}$ Examples include multifunctional sensors (e.g., temperature, strain, and electrophysiological), active/passive circuit elements (e.g., transistors, diodes, and resistors), wireless power coils, and components for radiofrequency communications (e.g., inductors, capacitors, oscillators, and antennas). Careful designs, guided by computational and analytical models of the mechanics, can yield platforms that are sufficiently compliant to laminate onto soft, complex curvilinear tissues. Effective physical characteristics such as thickness, area mass density, elastic modulus, thermal properties, and stretchability can match those of the targeted biological materials, such as the epidermis. The latter types of devices are sometimes referred as epidermal electronics. Figure 4a (left) shows a multifunctional example designed to allow measurement of electrophysiological (EP) signals generated by the human body. The device layout adopts an open, serpentine filamentary mesh design of active electronic materials (i.e., Si NM strain sensors, Si NM photovoltaics, InGaN light emitting diodes (LEDs), electrophysiological sensors and components for wireless communication systems) embedded in a thin, low modulus silicone elastomer. Figure $4 a$ (center) shows an image of such a device, peeling away from the forehead after use. Figure $4 a$ (right) presents electroencephalogram data collected during the closing and opening of the eyes. The data illustrate alpha rhythms that appear when the eyes are closed while the subject is awake. The signal quality compares favorably to that of data collected using conventional, bulk electrodes coupled to the scalp using conductive gels. Related examples are in operation on the trunk or limbs of the body, where electromyography (EMG) data associated with skeletal muscle contractions and electrical stimulation represent both sensing and actuating functionality. ${ }^{80}$

Figure 4b (left) shows a multifunctional epidermal electronic system with electrical stimulation electrodes and EMG, temperature, and strain sensors mounted on the skin. The device maintains its functionality without significant constraint on natural motions of the skin. As a demonstration of the stimulation capability, the devices interface to the long head of the biceps brachii and the lateral head of the triceps brachii muscles as shown in Fig. 4b (center). Flexing or extending the elbow joint generates EMG signals that allow control of a robot arm, with feedback through stimulation defined by coordinated operation of the two electrodes in Fig. $4 \mathrm{~b}$ (right). This result has relevance as a control interface to a prosthetic. The other possibility, in which similar devices can serve as sensory skins on robotic limbs, can also be considered. Figure $4 c$ (left $^{81}$ shows an image of an artificial skin that covers the surface of a prosthetic hand. The inset shows the mechanical stretchability. This platform includes Si NM strain, pressure and temperature sensor arrays, capable of detecting spatio-temporal distributions of strain associated with complex motions, in addition to thermal properties and ambient humidity. Figure $4 \mathrm{c}$ (right) summarizes the performance of Si NMs pressure sensors during typing a keyboard and catching a ball, as examples of the possibilities. 


\section{* Design Approaches}
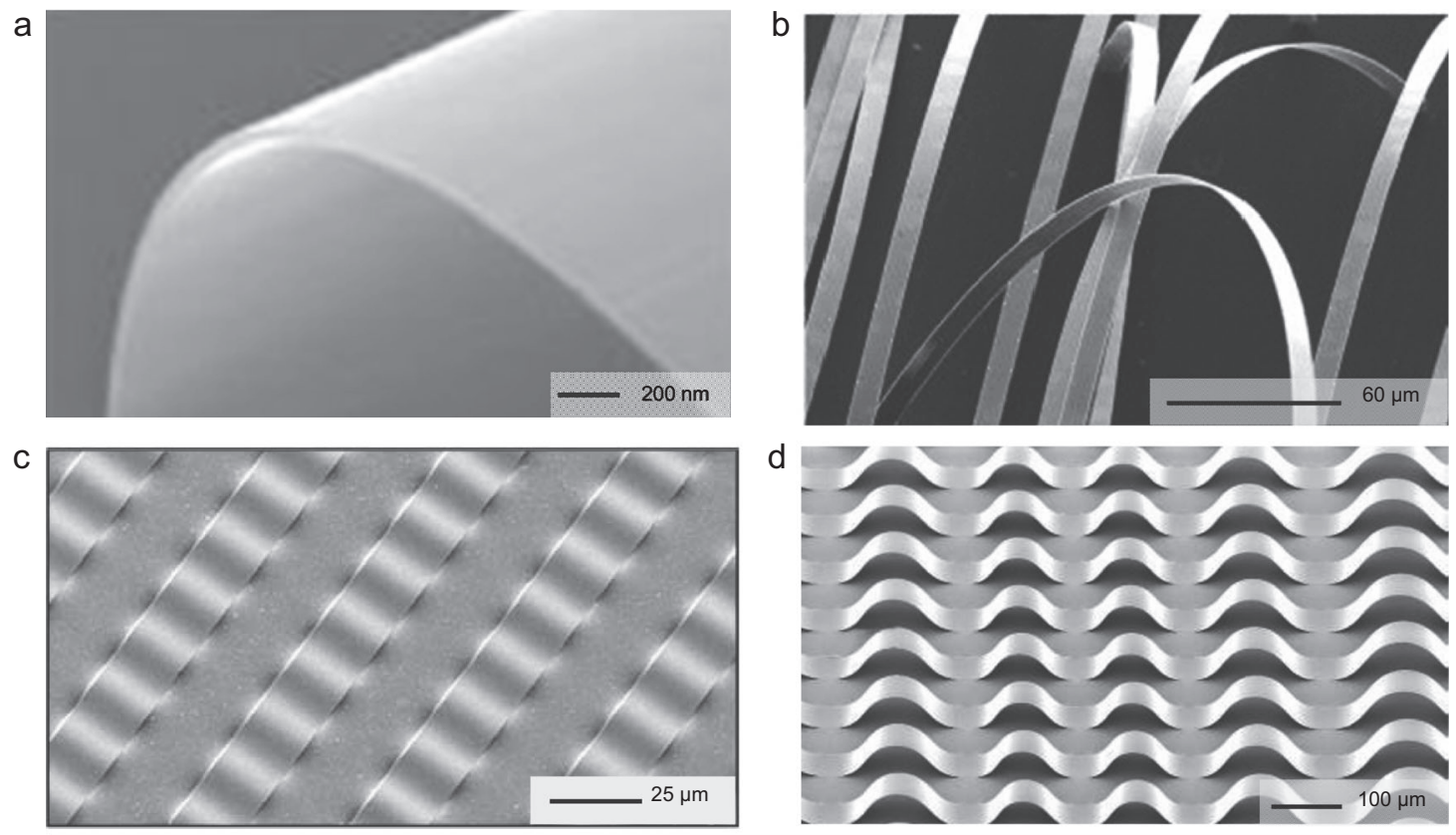

e

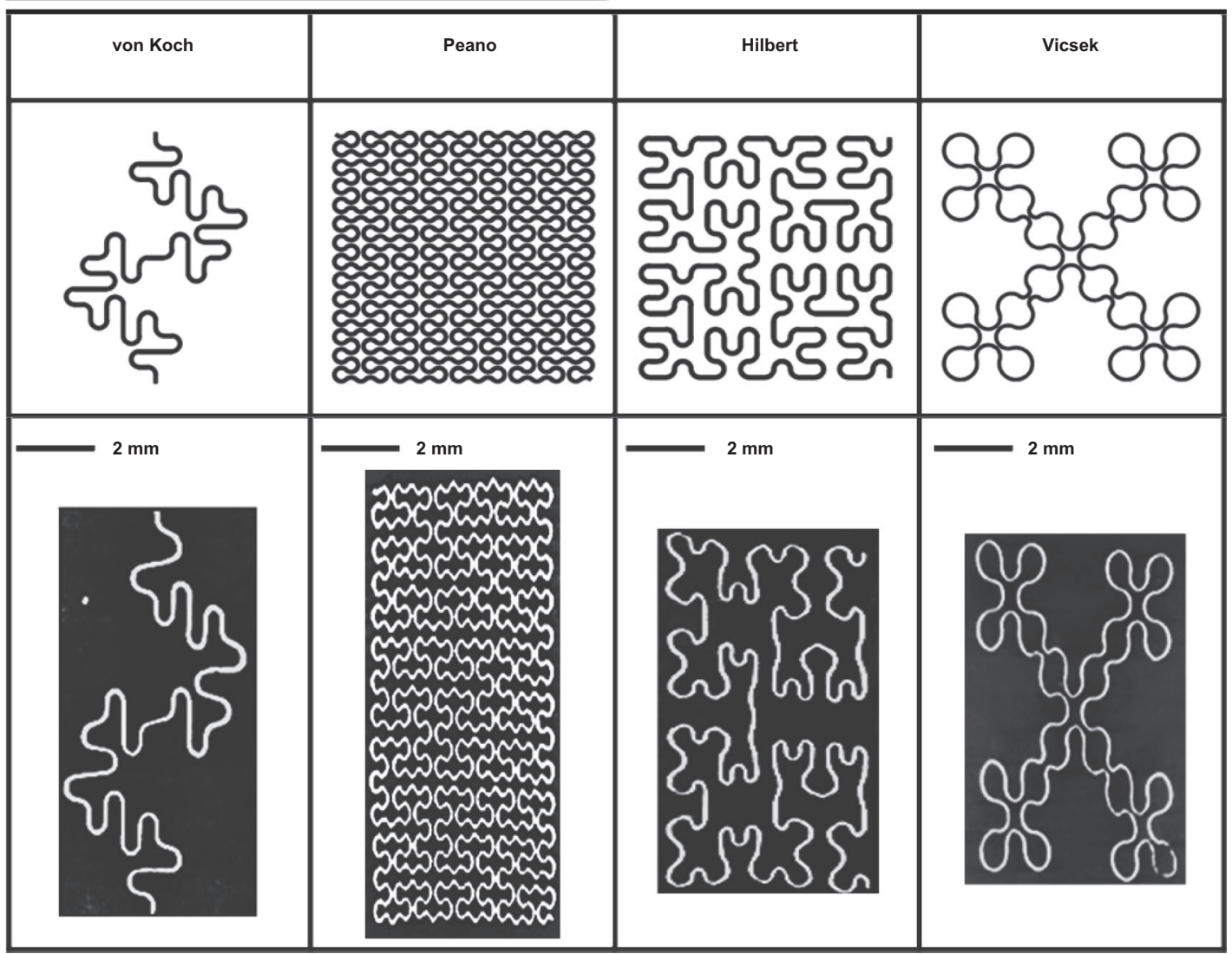

Fig. 3 Design approaches for flexible and stretchable electronics. a SEM images of a Si NM, to emphasize its high degree of mechanical flexibility, by virtue of its thin geometry. Reprinted with permission from Rogers et al. ${ }^{34}$ Copyright 2011 Nature Publishing Group. b Flexible Si NRs for transistors. Reprinted with permission from Mack et al. ${ }^{53}$ Copyright 2006 American Institute of Physics Journals. c Stretchable Si NRs, formed in a periodic wavy structure and bonded to an elastomer substrate. Reprinted with permission from Khang et al. ${ }^{76}$ Copyright 2006 American Association for the Advancement of Science. d Controlled buckling of Si NRs by lithographically defined adhesion sites to an underlying elastomer. Reprinted with permission from Sun et al. ${ }^{77}$ Copyright 2006 Nature Publishing Group. e Fractal serpentine design concepts for stretchable electronics including the design patterns (top) and corresponding MicroXCT images of the patterns after stretching. The uniaxial prestrains are $55 \%, 75 \%, 40 \%$, and $35 \%$ from left to right, respectively. Reprinted with permission from Fan et al. ${ }^{79}$ Copyright 2014 Nature Publishing Group 


\section{Epidermal Electronics 1}
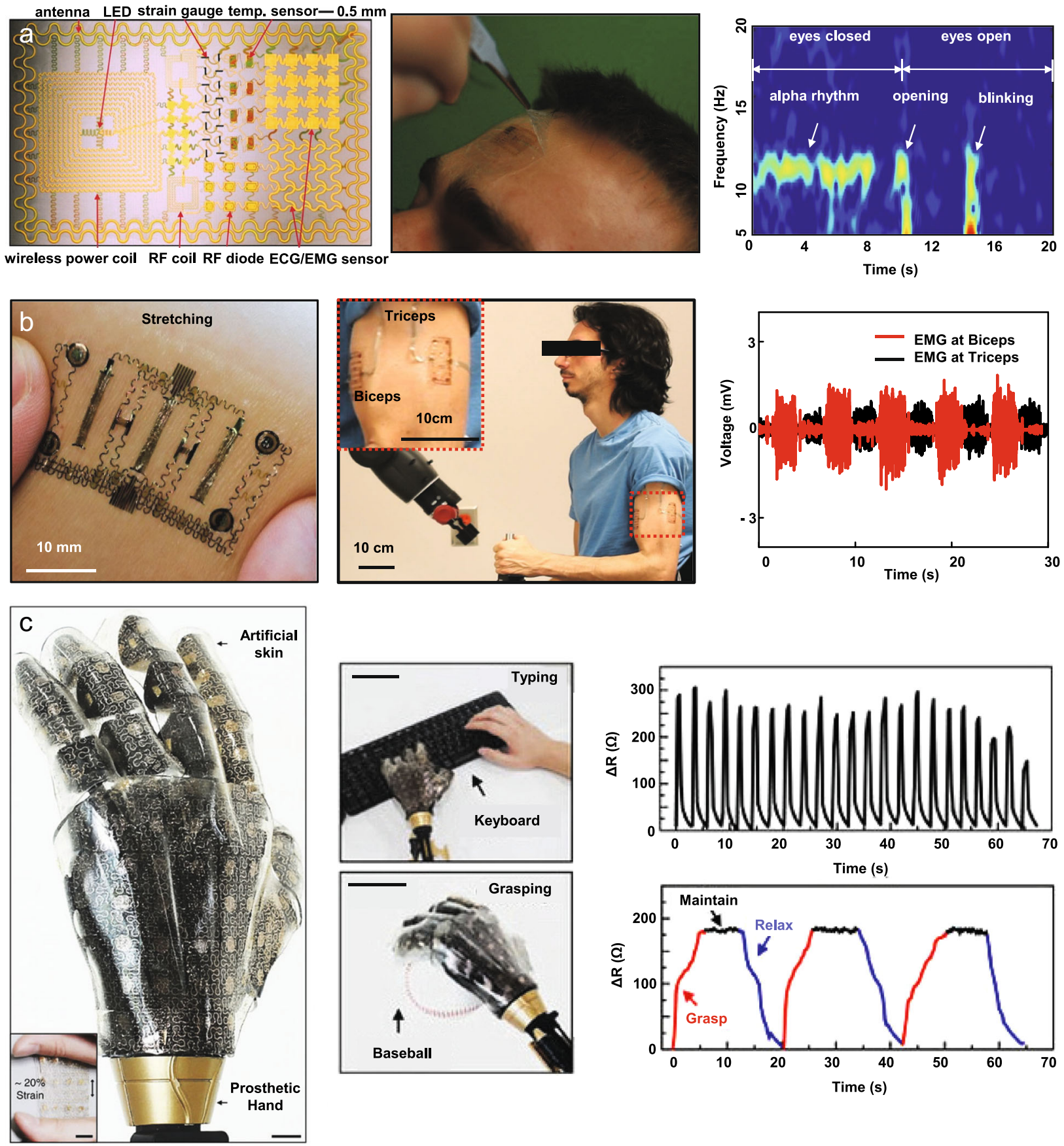

Fig. 4 Skin-like, or "epidermal", electronic system. a Image of a system with integrated electrodes, semiconductor components, and wireless devices in a multifunctional format (left). These systems can conformally laminate onto surface of the skin (center). The spectrograms show recorded EEG data that correspond to an awake subject when the eyes are closed and open. ${ }^{8}$ Reprinted with permission from Kim et al. ${ }^{8}$ Copyright 2011 American Association for the Advancement of Science. b Images a multifunctional epidermal device with capabilities in both sensing and stimulation, mounted on the forearm under stretching (left). Image of the devices on the bicep and tricep, for the purpose of controlling the angles of the elbow of a robotic arm (center). Corresponding EMG signals from two devices during alternation between flexion and extension of the elbow angle of the robotic arm. Reprinted with permission from Xu et al. ${ }^{80}$ Copyright 2016 WILEY-VCH. $\mathbf{c}$ Image of a stretchable prosthetic skin equipped with Si NM-based temperature, strain, and pressure sensor arrays (left). Examples of operation of the prosthetic limb: images of typing a keyboard and grasping a ball using prosthetic hand (center). Plots of corresponding responses of the SiNM pressure sensor (right). Reprinted with permission from Kim et al. ${ }^{81}$ Copyright 2014 Nature Publishing Group 
Most reported examples of these sorts of active electronic systems require connections to power supplies and/or external data acquisition systems. Hard wires provide one option, but wireless solutions are typically preferred. Extended versions of the design principles for wireless communications in epidermal electronics shown in Fig. 5 provide a solution. Commercial inorganic, chip-scale devices interconnected by thin-film metal traces serve as the basis for these systems. Additional device functions are possible by integrating other active components based on inorganic materials (e.g., Si and III-V semiconducting materials) onto soft polymer substrates as flexible backplanes. The system uses open mesh structures and fractal geometries for assemblies of customized chip-scale inorganic devices sealed within stretchable microfluidic chambers for recording EP signals with high-signal to noise ratio, multiplexed readout and wireless communication. Figure 5 a (left) ${ }^{82}$ shows an image of such a device powered by radio frequency energy transfer, in a twisted format. A completed device laminated onto the surface of the forearm in a deformed state appears in Fig. $5 a$ (center) ${ }^{82}$ The ability to measure EP signals using this device allows tether-free electrocardiography (ECG) recording, as in Fig. 5a (right). The data show clear $\mathrm{Q}, \mathrm{R}$, and $\mathrm{S}$ waveforms with high signal to noise ratio. Another example of battery-free, wireless epidermal electronics uses near-field communication technology (NFC) ${ }^{83,84}$ for multicolor light emission from LEDs and detection using photodetectors for measurements of various optical properties of skin. Such designs allow for monitoring of heart rate, tissue oxygenation, pressure pulse dynamics, UV exposure, and skin color. ${ }^{84}$ Figure $5 \mathrm{~b}$ (left) shows an image of an epidermal system equipped with red and IR LEDs mounted on the forearm for quantitative measurement of tissue oxygenation and its temporal variations, along with a schematic illustration of the operating principle (inset). Figure $5 \mathrm{~b}$ (center and right) shows the case of venous occlusion with the changes of total hemoglobin obtained using commercial oximeter and comparison to data from a wireless epidermal device. The results demonstrate the accuracy that can be achieved.

The most advanced example of this general type combines thin, miniaturized components for energy storage, power management, memory, physiological monitoring and wireless near-field communications, with capabilities in sensing, data logging and local processing. ${ }^{85}$ Figure $5 \mathrm{c}$ (left) shows an optical image of a device that includes a temperature sensor and a wireless data transmission module, along with data storage, power regulation of a battery array and wireless power delivery system. This platform is capable of storing information on temperature changes of the skin in the memory and wirelessly transmitting this data. A practical application appears in Fig. $5 c$ (center), with a batteryintegrated device (TI NFC) on the forearm of a subject on a stationary bike and a wirelessly powered NFC device without a battery module (AMS NFC) on an adjacent side of the arm as a point of comparison. Temperature responses from both the battery and wirelessly powered NFC devices are similar, as in Fig. 5c (upper right). The data are consistent with IR camera recordings in Fig. 5c (bottom right frames). Collectively, the results demonstrate feasibility in wireless epidermal electronics.

Other embodiments of the basic materials and design ideas allow radically different types of form factors and applications. For example, the same concepts allow construction of fully integrated optoelectronic systems that mount onto narrow polymer filaments, as "injectable" platforms that can be inserted into soft tissues, including the brain. Here, thin geometries not only enable flexibility but they also minimize tissue damage during insertion, as well as inflammation and scarring during use. Figure 6a (left) ${ }^{86}$ presents an exploded view schematic illustration of such a system that includes microscale InGaN LEDs ( $\mu$-ILEDs), an optical detector, along with thermal and EP sensors and actuators, mounted on a releasable injection microneedle designed to provide sufficient rigidity for insertion into targeted tissue. The functional units align and stack into a single unit, resulting in a flexible device that is capable of delivering electrophysiological and optoelectronic function to the depth of brain with wireless and untethered operation. This technology offers important functionality in optogenetics experiments, where the light emission can stimulate or inhibit the action of genetically targeted neurons. Tether-free operation provides many advantages compared to traditional approaches based on fiber optic cables and external light sources, due to enabling capabilities in studies of social interactions, activity in complex 3D environments and other circumstances that would lead to entanglement of the fiber optics. Wirelessly triggered optogenetic stimulus using these injected $\mu$-ILEDs allows control of complex neural circuitry in the brain, leading to specific behavioral responses that can serve as the basis for neuroscience studies. Figure $6 a$ (right) shows activity patterns of a mouse optogenetically trained to exhibit a place-preference in a Y-maze, as described in detail elsewhere. ${ }^{87}, 88$

Advanced designs in filamentary, stretchable antennas yield miniaturized designs and soft mechanics, suitable for full implantation, as depicted in Fig. $6 \mathrm{~b}$ (left). ${ }^{89}$ This antenna not only reduces by nearly two orders of magnitude the overall volume and weight of the complete system compared to originally reported designs, but it also supports broad bandwidth operation to enhance the reliability of operation. The soft interface allows implantation in locations where fixturing to hard, bony structures is not possible, such as underneath the leg muscles for optogenetic stimulation of a peripheral nerve, as in Fig. $6 \mathrm{~b}$ (center). Insertion into the epidural space as an interface to the spinal cord is also possible. See Fig. $6 c$ (right). The essential concepts in flexible, inorganic electronics underpin these and other new tools for biological research and clinical medicine.

\section{PHYSICALLY TRANSIENT ELECTRONICS}

Electronic materials in ultrathin layouts supported by unusual classes of substrates enable completely different opportunities in device characteristics, including but not confined by flexible/ stretchable mechanics. A particularly important example is in transient electronics, where a carefully selected set of constituent materials allows devices that are capable of high-performance operation over a user-defined period of time, after which they physically disappear, by controlled dissolution, depolymerization, micro-fragmentation or other mechanism. Such technologies have the potential to create new applications such as systems that resorb in the body, as temporary biomedical implants, or harmlessly disappear in the environment, as "green" electronics, or vanish in the field, for sensitive military hardware, or physically self-destruct, as a hardware-secure form of digital memory. Si NMs or Si NRs can serve as the foundational semiconductors because device-grade monocrystalline silicon has been recently revealed to be water-soluble, ${ }^{90}$ with biologically and environmentally benign end products, i.e., silicic acid and hydrogen. This realization immediately establishes routes to fully soluble high-performance active devices such as transistors, diodes, and other essential components of overall systems, when the Si NMs are combined with dissolvable metals, ${ }^{91}$ oxide dielectrics, ${ }^{92}$ and supporting biodegradable polymer substrates/encapsulants. ${ }^{93}$ As an example, Fig. 7a (left) ${ }^{94}$ presents a time sequence of atomic force micrographs of a Si NM collected at different stages of dissolution in phosphate-buffered saline (PBS; pH of 7.4) at $37^{\circ} \mathrm{C}$. Dissolution occurs in a continuous, predictable fashion with ultra-slow rates, to a large extent negligible for silicon in wafer form, but critically important for ultrathin geometries such as Si NMs (or NWs, etc). Dissolvable, biocompatible metals, such as $\mathrm{Mg}$, and dielectrics such as $\mathrm{MgO}$ or $\mathrm{SiO}_{2}$, and polymer substrates made of silk fibroin, for example, serve as the basis for diverse functional devices. Many other materials options are also available. ${ }^{91,95,} 96$ Figure $7 \mathrm{~b}$ shows images of a representative device platform that includes 


\section{Epidermal Electronics 2 (Wireless)}
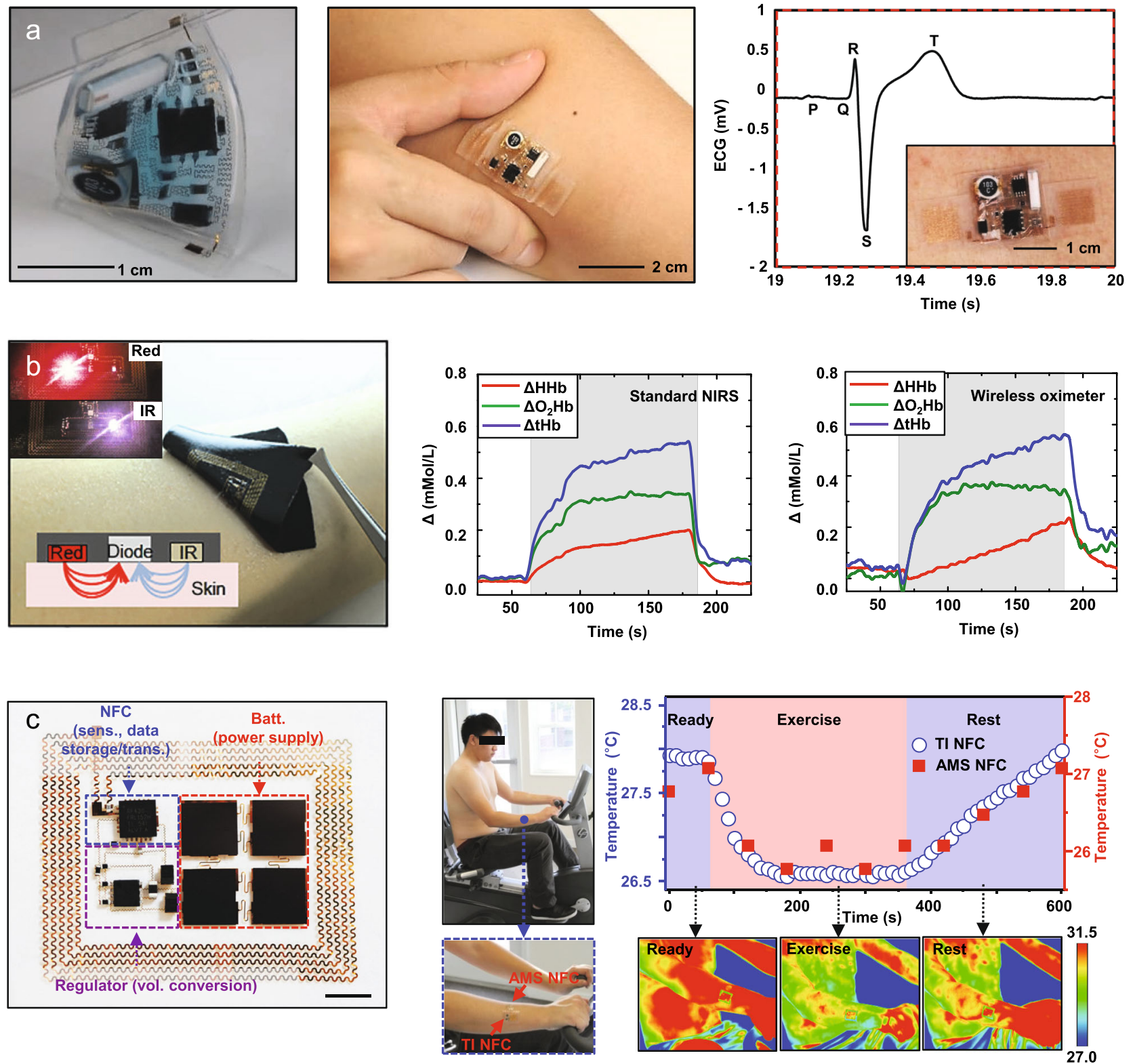

Fig. 5 Wirelessly powered epidermal electronic systems. a Images of a stretchable epidermal electronic system that includes interconnected commercial chips in a closed microfluidic space for a fully wireless EP sensor, with an example of the device on the forearm, in compressed and twisted states (center). Representative ECG data show the expected waveforms collected with the device integrated on the sternum. Reprinted with permission from Xu et al. ${ }^{82}$ Copyright 2014 American Association for the Advancement of Science. b Image of a wireless stretchable optoelectronic system mounted on the forearm and its operating principle (left). Time-domain near-IR (NIR) spectroscopy data simultaneously recorded by a commercially available oximetry device (center) and a wireless epidermal oximetry device (right) laminated onto the adjacent locations of forearm. Reprinted with permission from Kim et al. ${ }^{84}$ Copyright 2016 American Association for the Advancement of Science. c Image of a wireless epidermal battery-integrated system with temperature sensing/data processing (left). Images of the devices placed onto the forearm during exercise (center). Plots of temperature obtained by both an epidermal NFC device without a battery (AMS NFC) and a battery-integrated epidermal NFC device (TI NFC) (top right). Corresponding IR images show the responses from each stage of the evaluation (bottom right). Reprinted with permission from Lee et al. ${ }^{85}$ Copyright 2016 National Academy of Sciences

inductors, capacitors, resistors, diodes, transistors, and interconnects and encapsulants all integrated on silk substrate, where the key defining characteristics are high-performance operation with constituent materials that are completely water soluble to biologically and environmentally safe end products. Figure $7 \mathrm{~b}$ (bottom frames) illustrates the disintegration and dissolution of such a system in deionized water. The materials choices and the selected thicknesses, particularly of the encapsulating layers and substrates, define the functional lifetimes of the devices.

Compelling application opportunities exist in the form of "temporary", electronically active biomedical implants that provide important diagnostic and/or therapeutic function during the course of a transient biological event, such as a wound healing process. After this period, the devices disappear completely in the body, thereby eliminating unnecessary device load without the 


\section{* Implantable Wireless Optogenetics}
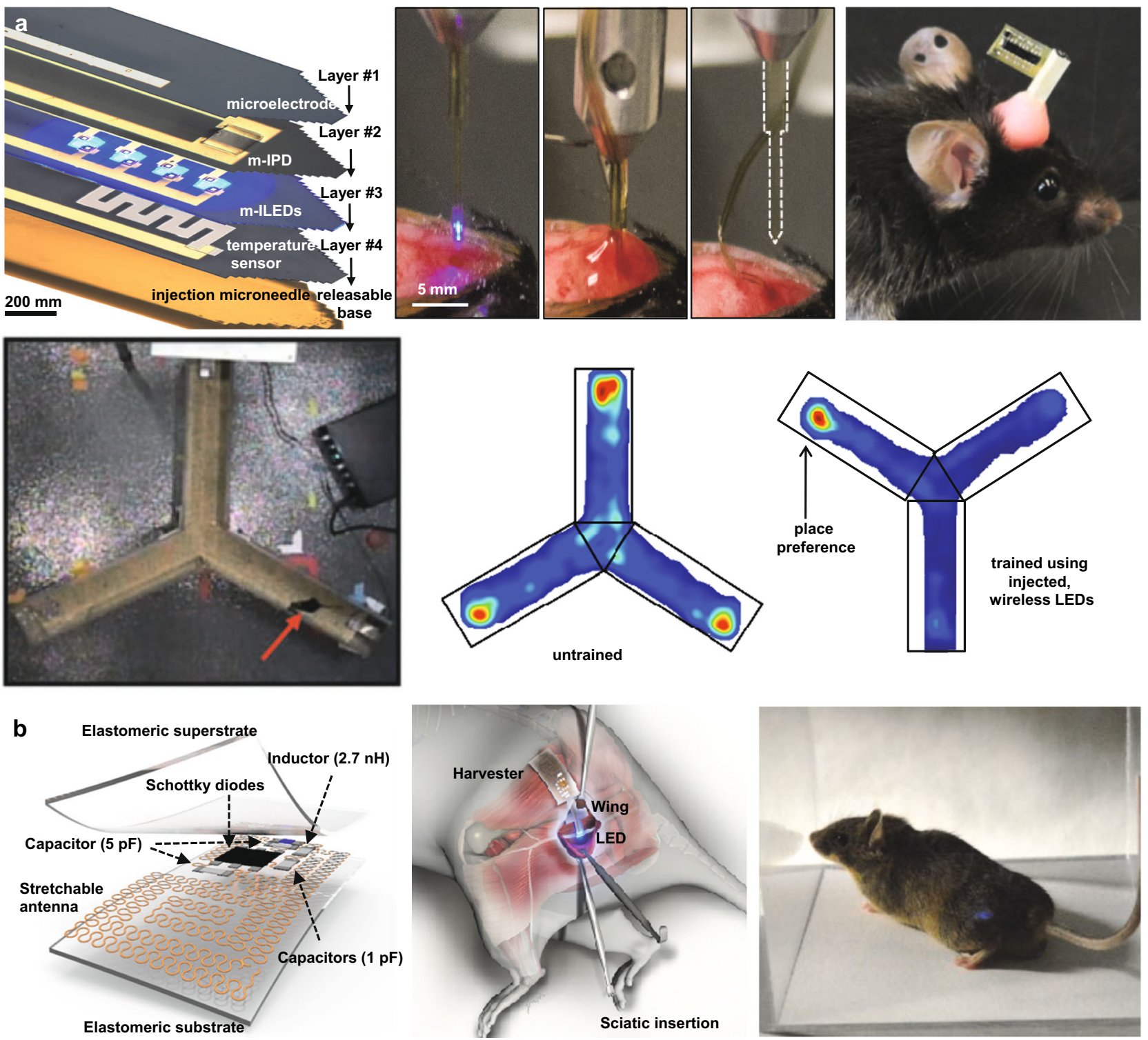

Fig. 6 Implantable wireless optogenetic system. a Exploded view images of a b multifunctional, injectable optoelectronic device that incorporates microelectrodes, Si NM photodetectors, inorganic microscale light emitting diodes ( $\mu$-ILEDs), and temperature and electrophysiological sensors integrated on a releasable injection microneedle (top left). Sequence of images of injection and release of the microneedle by dissolving a layer of silk as a temporary adhesive (top centers). Freely moving mouse with lightweight, flexible wireless optogenetic system. Heat maps of activity during motion in a Y-maze (bottom frames). Reprinted with permission from Kim et al. ${ }^{86}$ Copyright 2013 American Association for the Advancement of Science. b Exploded view schematic illustration of fully implantable stretchable wireless optogenetic device (left). The diagram shows the implanted wireless optogenetic device on the sciatic nerve (center). Image of a mouse with an optogenetic device implanted onto the sciatic nerve (right). Reprinted with permission from Park et al. ${ }^{89}$ Copyright 2015 Nature Publishing Group

need for a secondary surgery for device extraction. Figure $8 \mathrm{a}$ $(\text { left })^{32}$ highlights an exploded view and magnified image of a bioresorbable intracranial pressure monitor, of utility during a healing process following a severe traumatic brain injury. All of the constituent materials naturally resorb by hydrolysis in the cerebrospinal fluid after a defined operating period. Here, a piezoresistive strain gauge constructed with a doped Si NMs in a serpentine geometry lies near the edge of a suspended membrane of the bioresorbable polymer poly(lactic-glycolic acid) that is positioned above a etched trench in a bioresorbable substrate. External pressure deflects the membrane by an amount that can be inferred from the response of the strain gauge. Simple modifications of this basic design can support other kinds of function as well, including sensors for fluid, flow, motion, $\mathrm{pH}$ or thermal characteristics. Figure 8 a (center) shows an intracranial pressure sensor that uses a magnesium foil as a substrate, here configured to allow injection into the brain for monitoring pressure at a targeted depth. Figure 8 a (right) represents the intracranial pressure and temperature responses recorded using this type of bioresorbable sensor and a clinical standard, nonresorbable device. The results establish accuracy in measurement over a physiologically relevant range of pressures.

The same materials sets and concepts can enable bioresorbable devices for electronic monitoring as well. In an advanced example, 


\section{Transient Electronics 1}

a
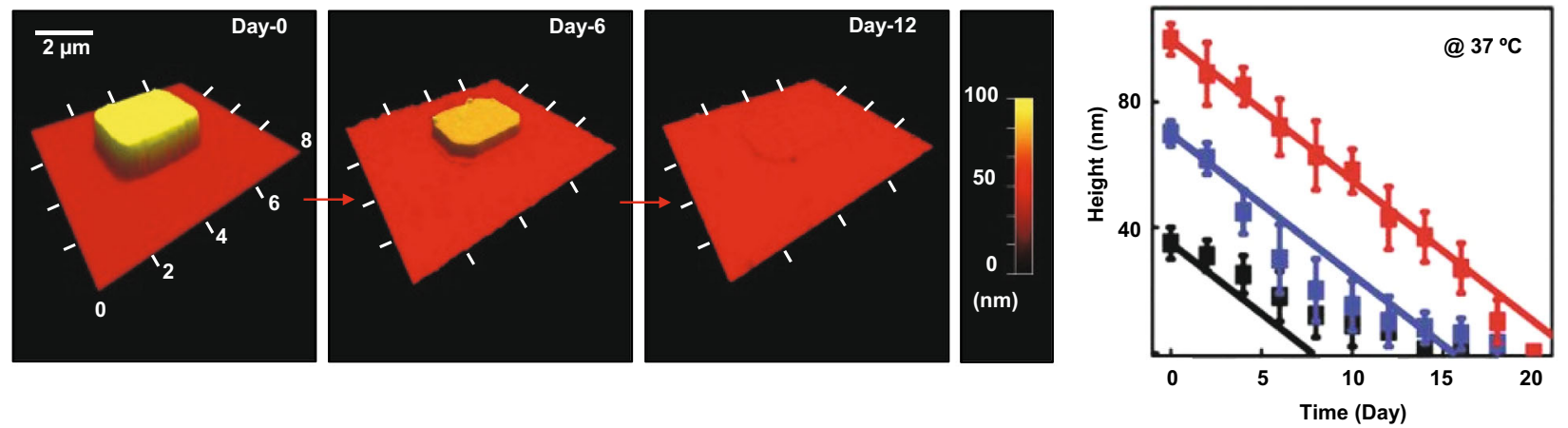

b
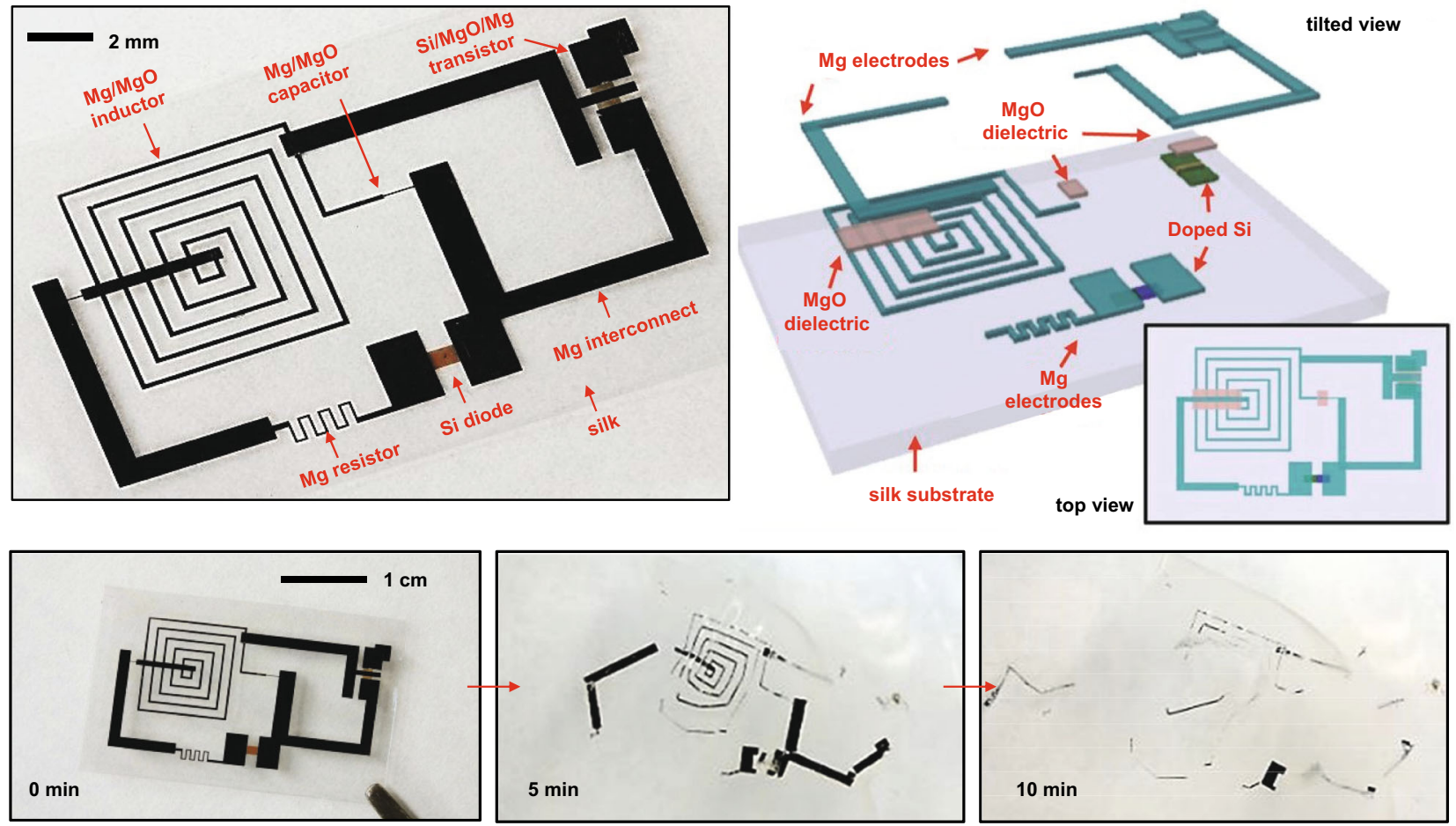

Fig. 7 Transient electronics. a Sequence of atomic force microscope images of a Si NM collected at different times of immersion in phosphatebuffered saline (PBS) at body temperature $\left(37^{\circ} \mathrm{C}\right.$ ) (left). Plot of dissolution of different thicknesses of Si NMs over time in PBS at body temperature obtained both theoretically and experimentally. $\mathbf{b}$ Image of a transient system with active device components integrated on a silk as a substrate (top left). Schematic exploded view illustration of a transient system (top right). Sequence of dissolution images of a transient system in PBS (bottom). Reprinted with permission from Hwang et al. ${ }^{94}$ Copyright 2012 American Association for the Advancement of Science

actively multiplexed sensor arrays in thin flexible formats allow mapping of electrical activity on the surface of the brain with spatial and temporal high resolution. ${ }^{97}$ Figure $8 \mathrm{~b}$ (left) shows a representative device that consist of 64 separate sensing electrodes and 24 wires as interfaces to external data acquisition systems. An important benefit of actively multiplexed readout, enabled by Si NM-based transistors at each electrode, is that it greatly reduces the number of wires needed for acquisition, which is particularly important at high channel counts. Figure $8 \mathrm{~b}$ (center) shows a cartoon and optical images of such a device laminated on the barrel cortex of the brain of a rodent model. The data in Fig. 8b (right, left column) show complex waveforms and delay plots that highlight clockwise spiral and diagonal spiral activities during an induced seizure. These examples illustrate excellent spatio-temporally resolved spiking activity, comparable to the best results possible with non-resorbable technologies (right, right column). ${ }^{10}$ These and other opportunities for bioresorbable electronic devices foreshadow even higher levels of sophistication and functional capabilities of relevance to many unmet clinical needs.

\section{CONCLUSION}

This review captures some of the most recent developments in high-performance flexible electronics based on single-crystalline inorganic semiconductor nanomaterials, with some emphasis on work from our group and system level demonstrations. These results, along with those highlighted in other related reviews, ${ }^{12,37,48,49,51,52}$ strongly suggest the potential of these ideas in unusual technologies with important characteristics that 


\section{Transient Electronics 2}

a

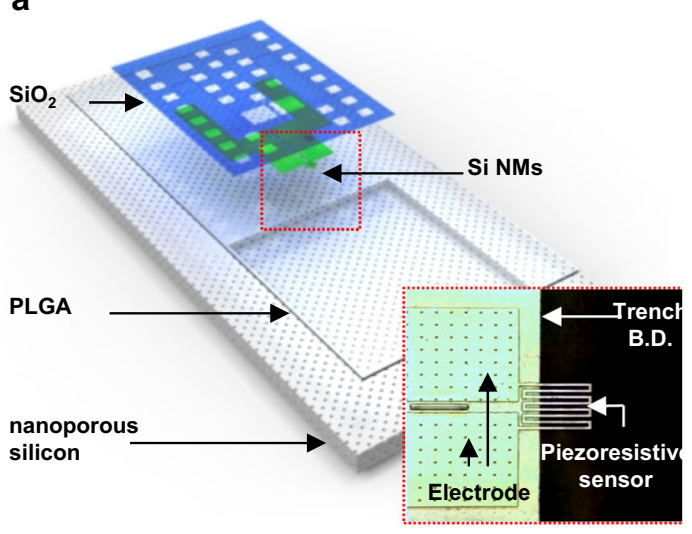

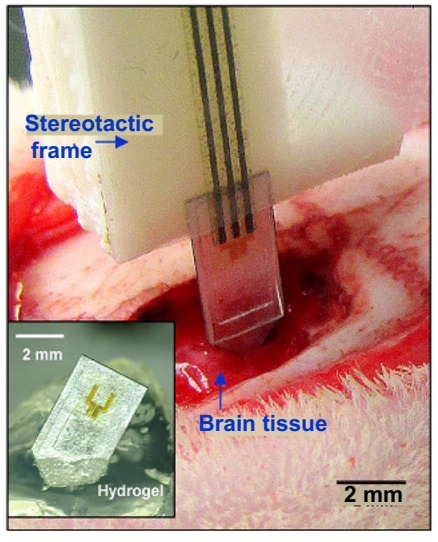

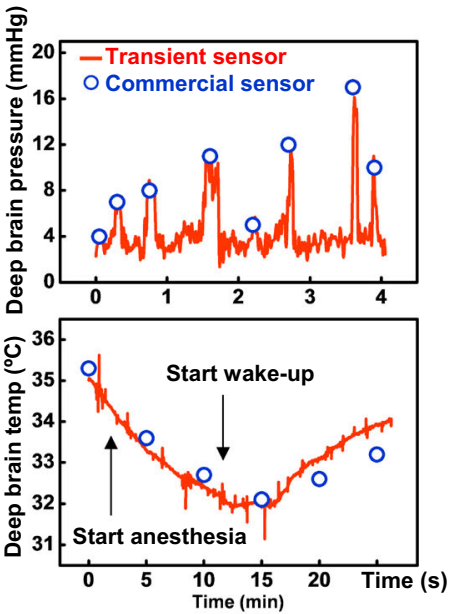

b

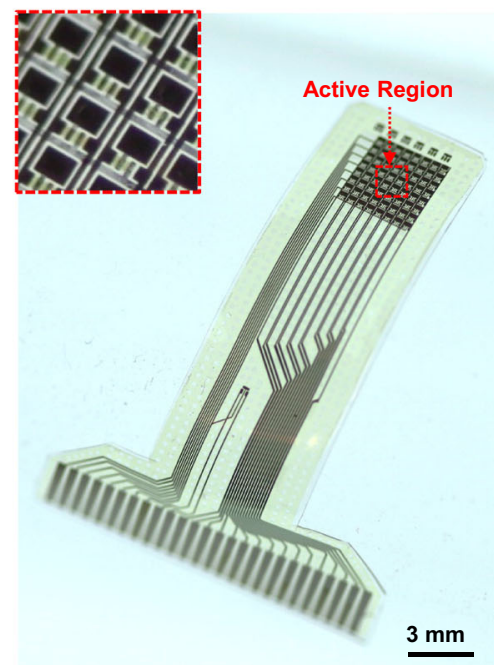

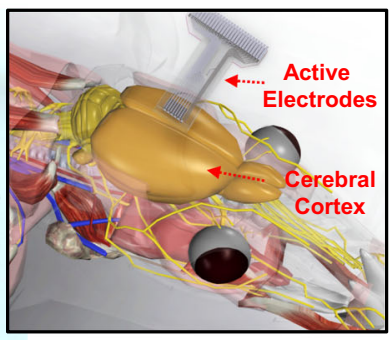

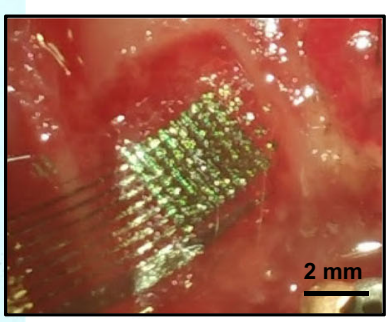

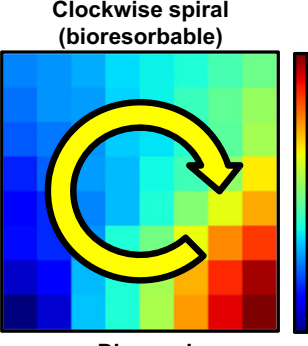

Diagonal

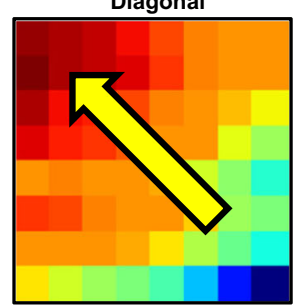

Counterclockwise spiral
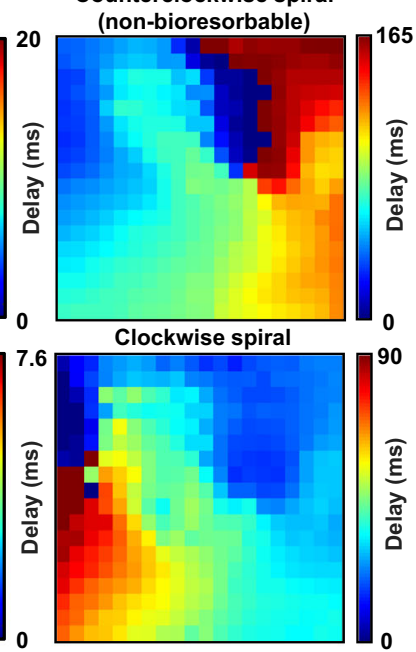

Fig. 8 Bioresorbable silicon electronics for the brain. a Schematic exploded view illustration of a bioresorbable sensor for intracranial pressure monitoring and optical image of the Si NM strain sensor, integrated at the edge of a cavity etched on the surface of a nanoporous silicon substrate (inset). Images of injection of a bioresorbable pressure senor for deep brain monitoring and of the device inserted into a hydrogel to mimic the brain environment (inset). Plots of in vivo measurements of pressure (top right) and temperature (bottom right) in the deep brain using a bioresorbable sensor and a commercial sensor as a point of comparison. Reprinted with permission from Kang et al. ${ }^{32}$ Copyright 2016 Nature Publishing Group. b Image of a 64-channel bioresorbable actively multiplexed array for electrocorticography (ECoG). Schematic illustration (top center) and optical (bottom center) images of the devices, mounted on the cerebral cortex of a rat brain. Delay map for the bandpass filtered data of epileptic spike activity (right). Reprinted with permission from Yu et al. ${ }^{97}$ Copyright 2016 Nature Publishing Group

cannot be achieved using conventional wafer-based electronic systems. Certain devices in skin-mounted wearables that exploit stretchable electronics are already now available commercially, at low cost and high volumes, ${ }^{98}, 99$ with many more to come. Advanced implants and surgical tools based on flexible/stretchable electronics are likely to appear next, with bio/eco resorbable systems not far after. These opportunities for broader impacts, combined with many interesting associated topics in fundamental materials science, mechanical and electrical engineering, create significant growth potential for this field of research.

\section{ACKNOWLEDGEMENTS}

We acknowledge the Department of Energy, the National Science Foundation and the Defense Advanced Research Projects Agency for financial support over the years.
This work was also supported (in part) by the Yonsei University Future-leading Research Initiative of 2017 (RMS2 2017-22-00).

\section{AUTHOR CONTRIBUTIONS}

K.J.Y., Z.Y., M.H., and J.A.R. collected the data, contributed to the scientific discussions, and co-wrote the manuscript.

\section{ADDITIONAL INFORMATION}

Competing interests: The authors declare no competing financial interests.

Publisher's note: Springer Nature remains neutral with regard to jurisdictional claims in published maps and institutional affiliations. 


\section{REFERENCES}

1. Zardetto, V., Brown, T. M., Reale, A. \& Di Carlo, A. Substrates for flexible electronics: A practical investigation on the electrical, film flexibility, optical, temperature, and solvent resistance properties. J. Polym. Sci. Part B: Polym. Phys. 49, 638-648 (2011).

2. Someya, T. et al. A large-area, flexible pressure sensor matrix with organic fieldeffect transistors for artificial skin applications. Proc. Natl Acad. Sci. USA 101, 9966-9970 (2004).

3. Yoon, J. et al. Ultrathin silicon solar microcells for semitransparent, mechanically flexible and microconcentrator module designs. Nat. Mater. 7, 907-915 (2008).

4. Ahn, J.-H. et al. Heterogeneous three-dimensional electronics by use of printed semiconductor nanomaterials. Science 314, 1754-1757 (2006).

5. Kaltenbrunner, M. et al. An ultra-lightweight design for imperceptible plastic electronics. Nature 499, 458-463 (2013).

6. Someya, T., Bao, Z. \& Malliaras, G. G. The rise of plastic bioelectronics. Nature 540, 379-385 (2016).

7. Kim, D.-H. et al. Stretchable and foldable silicon integrated circuits. Science 320, 507-511 (2008).

8. Kim, D.-H. et al. Epidermal electronics. Science 333, 838-843 (2011).

9. Kim, D.-H. et al. Materials and noncoplanar mesh designs for integrated circuits with linear elastic responses to extreme mechanical deformations. Proc. Nat Acad. Sci. 105, 18675-18680 (2008).

10. Viventi, J. et al. Flexible, foldable, actively multiplexed, high-density electrode array for mapping brain activity in vivo. Nat. Neurosci. 14, 1599-1605 (2011).

11. Yokota, T. et al. Ultraflexible organic photonic skin. Sci. Adv. 2, e1501856 (2016)

12. Rogers, J. A., Someya, T. \& Huang, Y. Materials and mechanics for stretchable electronics. Science 327, 1603-1607 (2010).

13. Sekitani, T. et al. Stretchable active-matrix organic light-emitting diode display using printable elastic conductors. Nat. Mater. 8, 494-499 (2009).

14. Sekitani, T., Zschieschang, U., Klauk, H. \& Someya, T. Flexible organic transistors and circuits with extreme bending stability. Nat. Mater. 9, 1015-1022 (2010).

15. Oh, J. Y. et al. Intrinsically stretchable and healable semiconducting polymer for organic transistors. Nature 539, 411-415 (2016).

16. Chortos, A. et al. Mechanically durable and highly stretchable transistors employing carbon nanotube semiconductor and electrodes. Adv. Mater. 28, 4441-4448 (2016)

17. Tee, B. C.-K. et al. A skin-inspired organic digital mechanoreceptor. Science $\mathbf{3 5 0}$, 313-316 (2015).

18. Jang, K.-l. et al. Rugged and breathable forms of stretchable electronics with adherent composite substrates for transcutaneous monitoring. Nat. Commun. $\mathbf{5}$, 4779 (2014).

19. Imani, S. et al. A wearable chemical-electrophysiological hybrid biosensing system for real-time health and fitness monitoring. Nat. Commun. 7, 11650 (2016).

20. Chen, J. \& Liu, C. T. Technology advances in flexible displays and substrates. IEEE Access 1, 150-158 (2013).

21. Kim, S. et al. Flexible displays: low-power flexible organic light-emitting diode display device (Adv. Mater. 31/2011). Adv. Mater. 23, 3475-3475 (2011).

22. Rogers, J. A. et al. Paper-like electronic displays: Large-area rubber-stamped plastic sheets of electronics and microencapsulated electrophoretic inks. Proc. Natl Acad. Sci. 98, 4835-4840 (2001).

23. Schwartz, G. et al. Flexible polymer transistors with high pressure sensitivity for application in electronic skin and health monitoring. Nat. Commun. 4, 1859 (2013).

24. Gao, W. et al. Fully integrated wearable sensor arrays for multiplexed in situ perspiration analysis. Nature 529, 509-514 (2016)

25. Lee, $\mathrm{H}$. et al. A graphene-based electrochemical device with thermoresponsive microneedles for diabetes monitoring and therapy. Nat. Nano 11, 566-572 (2016).

26. Koh, A. et al. A soft, wearable microfluidic device for the capture, storage, and colorimetric sensing of sweat. Sci. Transl. Med. 8, 366ra165-366ra165 (2016).

27. Vidor, F., Meyers, T. \& Hilleringmann, U. Flexible electronics: integration processes for organic and inorganic semiconductor-based thin-film transistors. Electronics 4, 480 (2015).

28. Fang, $\mathrm{H}$. et al. Ultrathin, transferred layers of thermally grown silicon dioxide as biofluid barriers for biointegrated flexible electronic systems. Proc. Natl Acad. Sci. 113, 11682-11687 (2016).

29. Lee, J.-S., Kovalenko, M. V., Huang, J., Chung, D. S. \& Talapin, D. V. Band-like transport, high electron mobility and high photoconductivity in all-inorganic nanocrystal arrays. Nat. Nano 6, 348-352 (2011).

30. Dutta, P. et al. High mobility single-crystalline-like GaAs thin films on inexpensive flexible metal substrates by metal-organic chemical vapor deposition. Appl. Phys. Lett. 105, 092104 (2014).

31. Kim, D.-H., Xiao, J., Song, J., Huang, Y. \& Rogers, J. A. Stretchable, curvilinear electronics based on inorganic materials. Adv. Mater. 22, 2108-2124 (2010).
32. Kang, S.-K. et al. Bioresorbable silicon electronic sensors for the brain. Nature 530, 71-76 (2016).

33. Park, S.-I. et al. Theoretical and experimental studies of bending of inorganic electronic materials on plastic substrates. Adv. Funct. Mater. 18, 2673-2684 (2008).

34. Rogers, J. A., Lagally, M. G. \& Nuzzo, R. G. Synthesis, assembly and applications of semiconductor nanomembranes. Nature 477, 45-53 (2011).

35. Pan, Z. W., Dai, Z. R. \& Wang, Z. L. Nanobelts of semiconducting oxides. Science 291, 1947-1949 (2001).

36. Kim, K. S. et al. Large-scale pattern growth of graphene films for stretchable transparent electrodes. Nature 457, 706-710 (2009).

37. Dasgupta, N. P. et al. 25th anniversary article: semiconductor nanowiressynthesis, characterization, and applications. Adv. Mater. 26, 2137-2184 (2014).

38. Kagan, C. R., Lifshitz, E., Sargent, E. H. \& Talapin, D. V. Building devices from colloidal quantum dots. Science 353, aac5523 (2016).

39. Tian, B. et al. Macroporous nanowire nanoelectronic scaffolds for synthetic tissues. Nat. Mater. 11, 986-994 (2012).

40. Liang, J. et al. Intrinsically stretchable and transparent thin-film transistors based on printable silver nanowires, carbon nanotubes and an elastomeric dielectric. Nat. Commun. 6, 7647 (2015).

41. Zhang, L. et al. A nanomesh scaffold for supramolecular nanowire optoelectronic devices. Nat. Nano 11, 900-906 (2016).

42. Yao, J., Yan, H. \& Lieber, C. M. A nanoscale combing technique for the large-scale assembly of highly aligned nanowires. Nat. Nano 8, 329-335 (2013).

43. Lu, W. \& Lieber, C. M. Nanoelectronics from the bottom up. Nat. Mater. 6 841-850 (2007)

44. Liu, Z., Xu, J., Chen, D. \& Shen, G. Flexible electronics based on inorganic nanowires. Chem. Soc. Rev. 44, 161-192 (2015).

45. Cheng, R. et al. Few-layer molybdenum disulfide transistors and circuits for highspeed flexible electronics. Nat. Commun. 5, 5143 (2014).

46. Wu, W. et al. Piezoelectricity of single-atomic-layer MoS2 for energy conversion and piezotronics. Nature 514, 470-474 (2014).

47. Fiori, G. et al. Electronics based on two-dimensional materials. Nat. Nano 9 768-779 (2014).

48. Kang, K. et al. High-mobility three-atom-thick semiconducting films with waferscale homogeneity. Nature 520, 656-660 (2015).

49. Song, J. et al. A general method for transferring graphene onto soft surfaces. Nat. Nano 8, 356-362 (2013).

50. Bae, S. et al. Roll-to-roll production of 30 -inch graphene films for transparent electrodes. Nat. Nano 5, 574-578 (2010).

51. Akinwande, D., Petrone, N. \& Hone, J. Two-dimensional flexible nanoelectronics. Nat. Commun. 5, 5678 (2014).

52. Chhowalla, M., Jena, D. \& Zhang, H. Two-dimensional semiconductors for transistors. Nat. Rev. Mater. 1, 16052 (2016)

53. Mack, S., Meitl, M. A., Baca, A. J., Zhu, Z.-T. \& Rogers, J. A. Mechanically flexible thin-film transistors that use ultrathin ribbons of silicon derived from bulk wafers. Appl. Phys. Lett. 88, 213101 (2006).

54. Baca, A. J. et al. Printable single-crystal silicon micro/nanoscale ribbons, platelets and bars generated from bulk wafers. Adv. Funct. Mater. 17, 3051-3062 (2007).

55. Ko, H. C., Baca, A. J. \& Rogers, J. A. Bulk quantities of single-crystal silicon micro-/ nanoribbons generated from bulk wafers. Nano Lett. 6, 2318-2324 (2006).

56. Huang, G. \& Mei, Y. Thinning and shaping solid films into functional and integrative nanomembranes. Adv. Mater. 24, 2517-2546 (2012).

57. Meitl, M. A. et al. Transfer printing by kinetic control of adhesion to an elastomeric stamp. Nat. Mater. 5, 33-38 (2006).

58. Lasky, J. B. Wafer bonding for silicon-on-insulator technologies. Appl. Phys. Lett. 48, 78-80 (1986).

59. Huang, M., Cavallo, F., Liu, F. \& Lagally, M. G. Nanomechanical architecture of semiconductor nanomembranes. Nanoscale 3, 96-120 (2011).

60. Colinge, J. P., Gao, M. H., Romano-Rodriguez, A., Maes, H. \& Claeys, C. in Technical Digest-International Electron Devices Meeting 595-598 (San Francisco, CA 1990).

61. Jang, $H$. et al. Quantum confinement effects in transferrable silicon nanomembranes and their applications on unusual substrates. Nano Lett. 13, 5600-5607 (2013)

62. Zhang, P. et al. Electronic transport in nanometre-scale silicon-on-insulator membranes. Nature 439, 703-706 (2006).

63. Roberts, M. M. et al. Elastically relaxed free-standing strained-silicon nanomembranes. Nat. Mater. 5, 388-393 (2006).

64. Cavallo, F. \& Lagally, M. G. Semiconductors turn soft: inorganic nanomembranes. Soft Matter 6, 439-455 (2010).

65. Yu, B., En, W. G., An, J. X. \& Riccobene, C. E. Method of fabrication of semiconductor-on-insulator (SOI) wafer having a Si/SiGe/Si active layer, US 6410371 
66. Yuan, H.-C. \& Ma, Z. Microwave thin-film transistors using Si nanomembranes on flexible polymer substrate. Appl. Phys. Lett. 89, 212105 (2006).

67. Sun, L. et al. $12-\mathrm{GHz}$ thin-film transistors on transferrable silicon nanomembranes for high-performance flexible electronics. Small 6, 2553-2557 (2010).

68. Guoxuan, Q., Hao-Chih, Y., George, K. C., Weidong, Z. \& Zhenqiang, M. Flexible microwave PIN diodes and switches employing transferrable single-crystal $\mathrm{Si}$ nanomembranes on plastic substrates. J. Phys. D Appl. Phys. 42, 234006 (2009).

69. Dash, W. C. \& Newman, R. Intrinsic optical absorption in single-crystal germanium and silicon at silicon at $77^{\circ} \mathrm{K}$ and $300^{\circ} \mathrm{K}$. Phys. Rev. 99, 1151-1155 (1955).

70. Yuan, H.-C. et al. Flexible photodetectors on plastic substrates by use of printing transferred single-crystal germanium membranes. Appl. Phys. Lett. 94, 013102 (2009).

71. Yoon, J. et al. GaAs photovoltaics and optoelectronics using releasable multilayer epitaxial assemblies. Nature 465, 329-333 (2010).

72. Ko, H. et al. Ultrathin compound semiconductor on insulator layers for highperformance nanoscale transistors. Nature 468, 286-289 (2010).

73. Mei, Y. et al. Versatile approach for integrative and functionalized tubes by strain engineering of nanomembranes on polymers. Adv. Mater. 20, 4085-4090 (2008).

74. Schmidt, O. G. \& Eberl, K. Nanotechnology: thin solid films roll up into nanotubes. Nature 410, 168-168 (2001).

75. Kim, D.-H. \& Rogers, J. A. Stretchable electronics: materials strategies and devices. Adv. Mater. 20, 4887-4892 (2008).

76. Khang, D.-Y., Jiang, H., Huang, Y. \& Rogers, J. A. A stretchable form of singlecrystal silicon for high-performance electronics on rubber substrates. Science 311, 208-212 (2006).

77. Sun, Y., Choi, W. M., Jiang, H., Huang, Y. Y. \& Rogers, J. A. Controlled buckling of semiconductor nanoribbons for stretchable electronics. Nat. Nano 1, 201-207 (2006).

78. Kim, D.-H. et al. Optimized structural designs for stretchable silicon integrated circuits. Small 5, 2841-2847 (2009).

79. Fan, J. A. et al. Fractal design concepts for stretchable electronics. Nat. Commun. 5, 3266 (2014)

80. $\mathrm{Xu}, \mathrm{B}$. et al. An epidermal stimulation and sensing platform for sensorimotor prosthetic control, management of lower back exertion, and electrical muscle activation. Adv. Mater. 28, 4462-4471 (2016).

81. Kim, J. et al. Stretchable silicon nanoribbon electronics for skin prosthesis. Nat. Commun. 5, 5747 (2014).

82. $\mathrm{Xu}, \mathrm{S}$. et al. Soft microfluidic assemblies of sensors, circuits, and radios for the skin. Science 344, 70-74 (2014).

83. Kim, J. et al. Miniaturized battery-free wireless systems for wearable pulse oximetry. Adv. Funct. Mater. 27, 1604373-1603280 (2017)

84. Kim, J. et al. Battery-free, stretchable optoelectronic systems for wireless optical characterization of the skin. Sci. Adv. 2, e1600418 (2016).

85. Lee, J. W. et al. Soft, thin skin-mounted power management systems and their use in wireless thermography. Proc. Natl Acad. Sci. 113, 6131-6136 (2016).

86. Kim, T.-i et al. Injectable, cellular-scale optoelectronics with applications for wireless optogenetics. Science 340, 211-216 (2013).

87. Wan, M. D. Y. et al. Postoperative impairment of cognitive function in ratsa possible role for cytokine-mediated inflammation in the Hippocampus. Anesthesiology 106, 436-443 (2007).
88. Shoji, H., Hagihara, H., Takao, K., Hattori, S. \& Miyakawa, T. T-maze forced alternation and left-right discrimination tasks for assessing working and reference memory in Mice. J. Vis. Exp.: JoVE 60, e3300 (2012).

89. Park, S. I. et al. Soft, stretchable, fully implantable miniaturized optoelectronic systems for wireless optogenetics. Nat. Biotech. 33, 1280-1286 (2015).

90. Yin, L., Bozler, C., Harburg, D. V., Omenetto, F. \& Rogers, J. A. Materials and fabrication sequences for water soluble silicon integrated circuits at the $90 \mathrm{~nm}$ node. Appl. Phys. Lett. 106, 014105 (2015).

91. Yin, L. et al. Dissolvable metals for transient electronics. Adv. Funct. Mater. 24, 645-658 (2014).

92. Kang, S.-K. et al. Dissolution behaviors and applications of silicon oxides and nitrides in transient electronics. Adv. Funct. Mater. 24, 4427-4434 (2014).

93. Hwang, S.-W. et al. High-performance biodegradable/transient electronics on biodegradable polymers. Adv. Mater. 26, 3905-3911 (2014).

94. Hwang, S.-W. et al. A physically transient form of silicon electronics. Science 337, 1640-1644 (2012).

95. Irimia-Vladu, M., Głowacki, E. D., Voss, G., Bauer, S. \& Sariciftci, N. S. Green and biodegradable electronics. Mater. Today 15, 340-346 (2012).

96. Irimia-Vladu, M. et al. Biocompatible and biodegradable materials for organic field-effect transistors. Adv. Funct. Mater. 20, 4069-4076 (2010).

97. $\mathrm{Yu}, \mathrm{K}$. J. et al. Bioresorbable silicon electronics for transient spatiotemporal mapping of electrical activity from the cerebral cortex. Nat. Mater. 15, 782-791 (2016).

98. Roche-Posay., L. My UV Patch, http://www.laroche-posay.us/my-uv-patch? gclid=CjwKEAiA17LDBRDElqOGq8vR7m8SJAA1AC0_FOF6jd10-1Z4z41wHTvuz2 UhoSfXympy5LVGxflhyRoC9-jw wcB (2016).

99. M.C. 10, BioStamp RC. https://www.mc10inc.com/our-products/biostamprc (2016).

100. Wu, W., Wen, X. \& Wang, Z. L. Taxel-addressable matrix of vertical-nanowire piezotronic transistors for active and adaptive tactile imaging. Science 340, 952-957 (2013).

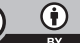

Open Access This article is licensed under a Creative Commons Attribution 4.0 International License, which permits use, sharing, adaptation, distribution and reproduction in any medium or format, as long as you give appropriate credit to the original author(s) and the source, provide a link to the Creative Commons license, and indicate if changes were made. The images or other third party material in this article are included in the article's Creative Commons license, unless indicated otherwise in a credit line to the material. If material is not included in the article's Creative Commons license and your intended use is not permitted by statutory regulation or exceeds the permitted use, you will need to obtain permission directly from the copyright holder. To view a copy of this license, visit http://creativecommons. org/licenses/by/4.0/.

(c) The Author(s) 2017 